\title{
Contents
}

1 Introduction 1

2 Types 3

2.1 Types are Sets of Classes . . . . . . . . . . . . . . . . . . 4

2.2 Subtyping is Set Inclusion . . . . . . . . . . . . . 5

2.3 Inheritance is Not Subtyping . . . . . . . . . . . . . . . 6

2.4 Other Type Systems . . . . . . . . . . . . . . 7

3 The Example Language $\quad 8$

3.1 Informal Semantics . . . . . . . . . . . . . . . . . 8

3.2 Static Correctness . . . . . . . . . . . . . . . . . . 10

3.3 Tree Representations of Classes . . . . . . . . . . . . . . . 12

3.4 Examples ...................... 14

4 Inheritance $\quad 15$

4.1 Syntax ........................... 15

4.2 Properties . . . . . . . . . . . . . . . . . . . 16

4.3 The Expansion Algorithm . . . . . . . . . . . . . . . 18

4.4 Example ...................... . . 22

5 Generalized Subclassing $\quad 25$

5.1 A Generalized Interpreter . . . . . . . . . . . . . 26

5.2 A Partial Order on Trees . . . . . . . . . . . . 26

5.3 Properties . . . . . . . . . . . . . . . . . . . . . . 29

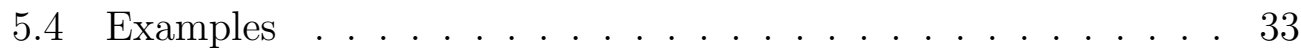


6 The Orthogonality Result 36

6.1 Two Suborders . . . . . . . . . . . . . . . 36

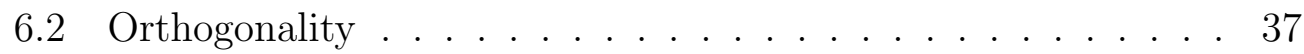

7 Class Substitution $\quad 40$

7.1 Syntax .......................... 40

7.2 Semantics . . . . . . . . . . . . . . . . 40

7.3 Pragmatics ...................... 42

8 Separate Compilation and Infinite Types 46

9 Conclusion $\quad 48$ 


\section{Static Typing for Object-Oriented Programming}

\author{
Jens Palsberg \\ palsberg@daimi.aau.dk
}

\author{
Michael I. Schwartzbach \\ mis@daimi.aau.dk
}

\author{
Computer Science Department \\ Aarhus University \\ Ny Munkegade \\ DK-8000 Arhus C, Denmark \\ Ny Munkegade
}

, Denmark

June 1991

\section{Introduction}

Object-oriented programming is becoming widespread. Numerous programming languages supporting object-oriented concepts are in use, and theories about object-oriented design and implementation are being developed and applied $[21,3,13]$. 
An important issue in object-oriented programming is to obtain reusable software components [30]. This is achieved through the notions of object, class, inheritance, late binding, and the imperative constructs of variables and assignments. Such features are found for example in the SMALLTALK language [25] in which a large number of reusable classes has been written. SMALLTALK, however, is untyped. Though this is ideal for prototyping and exploratory development, a static type system is required to ensure that programs are readable, reliable, and efficient.

This paper studies an idealized subset of SMALLTALK, which we equip with a novel static type system. Our analysis results in the definition of a new genericity mechanism, called class substitution, which we prove to be the order-theoretic complement of inheritance.

In the following section we briefly review the benefits of static typing. We argue that the choice of finite sets of classes as types can provide exactly those benefits. Furthermore, in this setting subtyping can be seen to be simply set inclusion. Finally, we compare this notion of type with others that have been proposed.

In section 3 we outline the example language. It is an idealized subset of SMALLTALK, in which variables and parameters are declared together with types. The language has been simplified by the omission of blocks; instead, a primitive if-then-else is provided. We also give precise requirements for static correctness of programs, and introduce a mathematical framework in which we represent classes as labeled, regular trees. These representations abstract away from class names.

In section 4 we discuss inheritance and its interaction with mutually recursive classes. We also show that a program using inheritance can be transformed into one which does not.

In section 5 we structure the class representations with a partial order which generalizes inheritance. The intuition behind the order is that a subclass may extend the implementation and redefine the types consistently, while preserving the recursive structure of the superclass. All such generalized subclass relationships can be exploited by an implementation to provide reusable software components. The suggested implementation is a generalization of the Smalltalk interpreter. We show that the partial order has the same characteristic properties as its subset inheritance: it is decidable, has a least 
element, has finite intervals, does not allow temporal cycles, and preserves subtyping.

In section 6 we prove that the generalized subclassing order is strictly more powerful than ordinary inheritance. We characterize the extra possibilities by showing that they form a suborder which is an order-theoretic orthogonal complement to the suborder formed by inheritance relationships.

In section 7 we develop the orthogonal complement of inheritance into a programming mechanism, called class substitution, which turns out to be a genericity mechanism. This means that our simple type system, though voided of e.g. type variables, still supports genericity. We extend the example language with syntax for class substitution, give example programs, and compare with parameterized classes.

Finally, in section 8 we use our framework to analyze the kind of type system which is common in existing object-oriented languages, for example $\mathrm{C}++$ and Simula/Beta. The analysis yields an explanation of why these languages often allow loop-holes in the type system or resort to run-time type-checking in some cases.

\section{Types}

Types are introduced into untyped languages because an untyped program may be unreadable, unreliable, and inefficient. Any choice of type system for a language must be able to remedy some or all of the above deficiencies [29].

Types may be used as annotations, and those can be read not only by humans but also by the compiler which may be able to exhibit a safety-guarantee and perform compile-time optimizations. The safety-guarantee will typically state that operations are only performed on arguments of the proper types; in other words, certain run-time errors will not occur.

In this section we present a new, simple type system for object-oriented languages and we argue why it yields the benefits stated above. We also examine other type systems and discuss similarities and differences. 


\subsection{Types are Sets of Classes}

The basic metaphor in object-oriented programming is the object. An object groups together variables and procedures (called methods), and prevents direct outside access to the variables; it may be thought of as a module [57]. Objects are instances of classes, see for example figure 1. The class Record specifies a pattern from which all Record objects will be created. Such an object is created for example in class File by the expression "Record new" and it gets a separate copy of all variables. Note that also Integer is a class, though specified elsewhere, and that each method returns the result of its last expression. If nothing needs to be returned, then usually one returns the object itself, denoted by self.

\begin{tabular}{|l} 
class Record \\
var key: Integer \\
method getKey returns Integer \\
key \\
method setKey(k: Integer) returns Record \\
key := $\mathrm{k}$; self \\
end Record \\
class File \\
var buffer: Record \\
method initialize returns File \\
buffer := Record new ; buffer.setKey $(17) ;$ self \\
end File
\end{tabular}

Figure 1: Records and Files.

The only possible interaction with an object is when sending a message to itwhen invoking one of its methods. For example, in class File the expression buffer.setKey(17) expresses the sending of the message setKey with argument 17 to the object in the variable buffer. If the object does not understand the message - if its class does not implement a method with the specified namethen the run-time error messageNotUnderstood occurs. In a pure objectoriented language this is the only run-time error that can occur.

The purpose of a type system is to allow the programmer to annotate programs with information about which methods are available in a given object, 
and to allow the compiler to guarantee the the error messageNotUnderstood will never occur $[4,8]$. The latter immediately enables compile-time optimizations because a number of checks need not be inserted into the code.

In SMalltalk, any object can be assigned to any variable. Message sending is implemented by late binding, i.e., the message send is dynamically bound to an implementation depending on the class of the receiver. The fundamental question is: which messages will an object residing in a variable be able to respond to. Ignoring the possibility of doing flow analysis, the answer is: those methods that are common to the classes of the possible objects in that variable. This set of methods can be specified by simply listing the classes of the possible objects, because only finitely many classes occur in a given program. These observations lead us to define the notion of type that will be analyzed throughout this paper.

$$
\text { A type is a finite set of classes. }
$$

Note that a type can only contain classes that are part of the program. This corresponds to a "closed-world" assumption.

Our types are more general than they may seem at first. Any kind of type expressions may be employed, providing that they can be interpreted as predicates on classes. In a given program only finitely many classes will satisfy any given predicate. The type can now be identified with the finite set; hence, we are justified in viewing our type system as being quite general.

As an example of a type, consider again figure 1, where class File specifies a variable buffer of type Record. The type contains a single class, hence, only Record objects are allowed to reside in the variable.

\subsection{Subtyping is Set Inclusion}

An object may have more than one type. Consider for example an instance of class Record. The singleton type containing just Record is a type of that instance, but so is also any superset. Henceforth, we will call a superset for a supertype, and a subset for a subtype.

When type-checking an assignment $x:=e$ (and similarly for a parameter passing), then it suffices to check that the static type of $x$ is a supertype of 
the static type of e. This is because the assignment then will not violate the requirement that only objects of the static type of $x$ can reside in $x$.

If we had a more advanced kind of type expressions, then subtyping must be defined to coincide with - or at least to respect - inclusion of the corresponding sets.

The nil object requires special attention. In SMALLTALK it is an instance of the class undefinedobject, and it is the initial contents of variables when objects are created. This implies that we should want undefinedObject to be a member of all types. In this paper, we do not want to rely on any predefined classes, however, so we will treat nil in another way. Instead of having undefinedObject as an explicit member of all types, we will define nil to be a special primitive value which implicitly has the empty type and, hence, has all types. Treating nil as a non-instance is in line with its implementation in typed languages such as $\mathrm{C}++$ [53], Eiffel [39], and Simula [22]/Beta [32]. In the language we later present, nil is in fact the only constant value. The language can be viewed as simply a calculus of pointers, in which it is quite natural that nil should enjoy a special status.

\subsection{Inheritance is Not Subtyping}

Object-oriented programming differs from other programming paradigms in offering inheritance as a means for reusing class definitions. Inheritance can be viewed as a shorthand: it allows the definition of a class to be a modification of an existing one. More specifically, it allows the construction of subclasses by adding variables and methods, and by overriding method bodies $[25,56]$. A thorough discussion of inheritance is given in a later section.

The difference between inheritance and subtyping is illustrated in figure 2 which displays a class hierarchy discussed in [37], together with the corresponding type hierarchy. Notice that we turn the class hierarchy "upsidedown" in order to get the smallest class at the bottom, and that the figure uses the notation $\uparrow C$ for the set of all subclasses of $C$, even potential ones. The class hierarchy is a tree, whereas the type hierarchy is a lattice.

Note that the BETA group interprets nil as an instance of an auxiliary class on top of the class hierarchy [37]. This is awkward because it implies that 


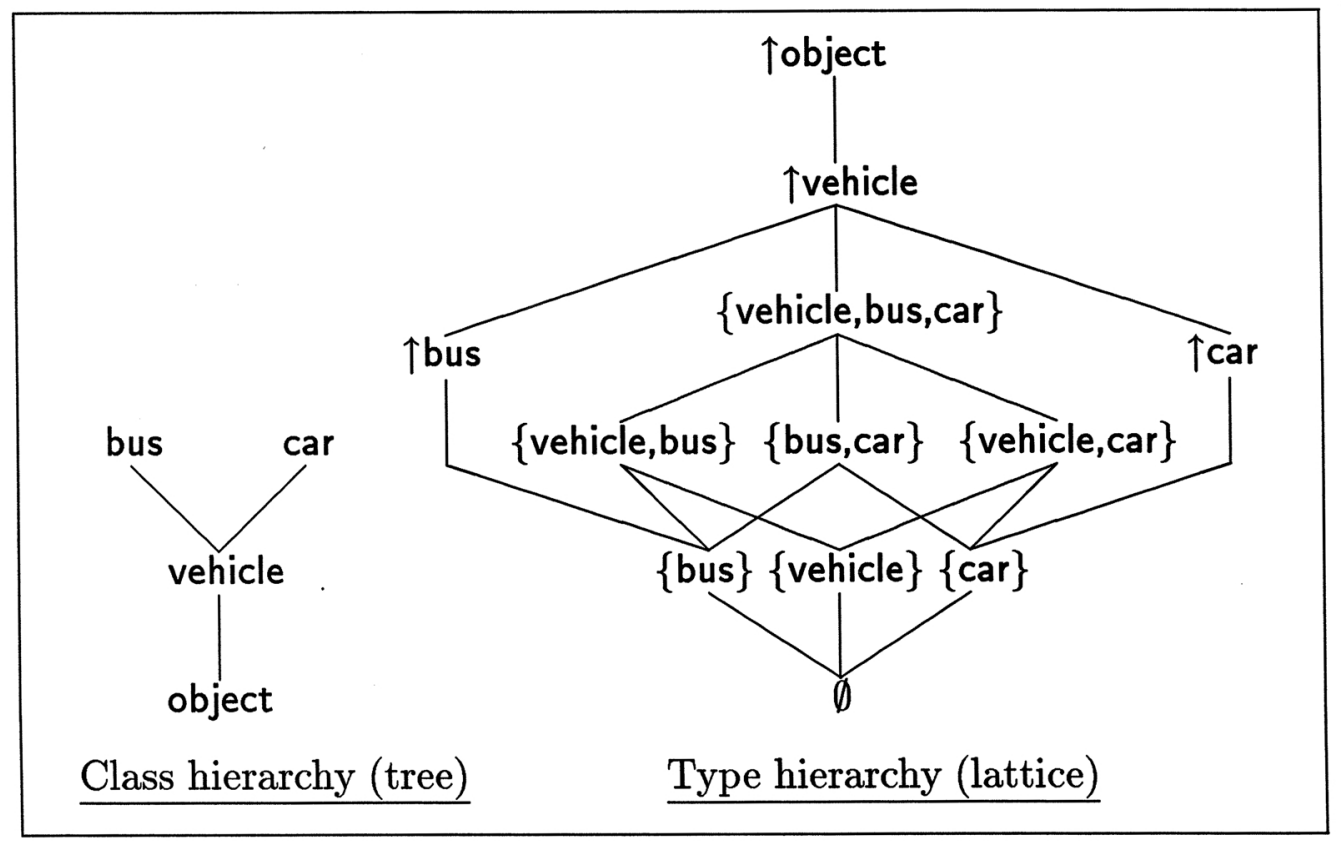

Figure 2: The class and type hierarchy (excerpts).

this class can be obtained by some sort of multiple inheritance of all other classes. This again implies that instances of this auxiliary class should be able to respond to any message; clearly nil is not able to do this. Our explanation of nil as a value having the empty type is more satisfactory: it reflects that nil can not respond to all messages, and that it can be assigned to any variable.

\subsection{Other Type Systems}

Usually, formal models of typed object-oriented programming are based on the lambda calculus. They represent objects as records and methods as functions, and involve coercions together with subtypes [9, 41], polymorphic types [40, 10, 23], or $F$-bounded constraints [18, 16, 7] in the description of inheritance. In contrast, traditional object-oriented languages are not based on coercions and do not support methods as values.

Furthermore, the coercion models - while being very general in some respectsdo not support variables and assignments, because variable (mutable) types 
have no non-trivial subtypes, as observed by Cardelli [11]. In a functional language, an assignment must be emulated by the creation of an updated copy, and it is extremely hard to preserve the type of the original value. It was long believed that bounded parametric polymorphism was sufficient [10], but it has been realized that considerably more fine-grained type systems are required to handle even simple updates [12].

Graver and Johnson's type system for SMALLTALK [27, 28] has much in common with ours. Their types are essentially finite sets of classes, but they have to axiomatize a subtype relation that corresponds exactly to set inclusion because the type system involves type variables. They also employ a notion of signature type which essentially denotes the finite set of subclasses of a given class, under a "closed-world" assumption.

One strength of our type system is that it can avoid type variables and exclusively use the simple notion of sets of classes as types. The issue of genericity will instead be handled by generalizing the notion of subclassing.

\section{The Example Language}

Our example language is an idealized subset of Smalltalk, except that variables and parameters are declared together with a type, see figure 3 . We also leave the issue of inheritance to the following section. In this section we briefly discuss the semantics of the language and state the precise rules for static correctness. We also introduce a convenient representation of classes.

\subsection{Informal Semantics}

A program is a set of classes followed by an expression whose value is the result of executing the program. A class can contain variables and methods; a method consists of a name, an argument-list, a result-type, and an expression. The result of an assignment is the assigned value. A message send is executed by evaluating the receiver and the arguments, and if the class of the receiver implements a method for the message, then a normal procedure call takes place; otherwise, the error messageNotUnderstood occurs. The result of a sequence is the result of the last expression in that sequence. The if-then-else 


\begin{tabular}{|c|c|}
\hline (Program) & $\mathrm{P}::=\mathrm{C}_{1} \ldots \mathrm{C}_{n} \mathrm{E}$ \\
\hline (Class) & $\begin{aligned} \mathrm{C}::= & \text { class ClassId } \\
& \text { var } \mathrm{D}_{1} \ldots \mathrm{D}_{k} \mathrm{M}_{1} \ldots \mathrm{M}_{n} \\
& \text { end ClassId }\end{aligned}$ \\
\hline $\begin{array}{l}\text { (Method) } \\
\text { (Declaration) }\end{array}$ & $\begin{array}{l}\mathrm{M}::=\text { method MethodId }\left(\mathrm{D}_{1} \ldots \mathrm{D}_{k}\right) \text { returnes } \mathrm{T} E \\
\mathrm{D}::=\mathrm{Id}: \mathrm{T}\end{array}$ \\
\hline (Type) & $\mathrm{T}::=[$ selfClass $]$ ClassId $_{1} \ldots$ ClassId $_{n}$ \\
\hline (Expression) & $\begin{aligned} \mathrm{E}::= & \mathrm{Id}:=\mathrm{E} \mid \mathrm{E} . \text { MethodId }\left(\mathrm{E}_{1} \ldots \mathrm{E}_{n}|\mathrm{E} ; \mathrm{E}|\right. \\
& \text { if } \mathrm{E} \text { then E else } \mathrm{E} \mid \text { ClassId new } \mid \text { selfClass new } \\
& \mathrm{E} \text { instancdOf ClassId } \mid \text { self }|\mathrm{Id}| \text { nil }\end{aligned}$ \\
\hline
\end{tabular}

Figure 3: Syntax of the example language.

expression tests if the condition is non-nil. The expression "selfClass new" yields an instance of the class of self. The expression "E instanceof ClassId" yields a run-time check for class membership. If the check fails, then the expression evaluates to nil. The expression self denotes the receiver of the message, Id refers to instance variables and parameters, and nil is a primitive value.

Note that selfClass always can be replaced by the name of the enclosing class. This is a convenient way of making recursion explicit. It is not sufficient for expressing mutually recursive classes, however. For that, it is necessary to use the class names directly.

We have no primitive types (like integer and boolean) nor type constructors (like list) because we can program classes that encode them, see [44].

Note that we ignore the issues of concurrency and persistence [55]. 


\subsection{Static Correctness}

We define correctness of a method body with respect to the name of the enclosing class, and global and local environments. These can be uniquely determined from the program syntax.

The global environment maps class names to class descriptions. A class description maps method names to method descriptions. A method description maps argument numbers to their declared types; for convenience, the result type is assumed to have number zero.

The local environment is a finite map from names of instance variables and parameters to their declared types.

We shall use the notation

$$
\text { E }:: \tau
$$

to denote that the expression $\mathrm{E}$ is statically correct and has type $\tau$. This property will be defined by means of a number of rules of the form

- Condition $_{1}$

$\vdots$

Condition $_{k}$

$\mathrm{E}:: \tau$

with the obvious interpretation: if all the conditions can be satisfied, then the conclusion follows.

The following rules exhaust the syntactic possibilities; the enclosing class has name $\mathrm{C}$, the global environment is denoted by $\mathcal{G}$, and the local environment is denoted by $\mathcal{E}$.

- nil ::\{\}

- self :: C

- selfclass new :: $\{C\}$ 
- D new :: $\{\mathrm{D}\}$

- id $\in \operatorname{dom}(\mathcal{E})$

early check Id $:: \mathcal{E}($ Id $)$

- E :: $\tau$

E instanceOf $D::\{D\}$

- $\mathrm{E}_{i}:: \tau_{i}$

if $\mathrm{E}_{1}$ then $\mathrm{E}_{2}$ else $\mathrm{E}_{3}:: \tau_{2} \cup \tau_{3}$

- $\mathrm{E}_{i}:: \tau_{i}$

$\mathrm{E}_{1} ; \mathrm{E}_{2}:: \tau_{2}$

- $\mathbf{E}:: \tau_{1}$

Id $:: \tau_{2}$

$\tau_{1} \subseteq \tau_{2}$

$\mathrm{Id}:=\mathrm{E}:: \tau_{1}$

subtype check

- E :: $\tau$

$\mathrm{E}_{i}:: \tau_{i}$

$\forall c \in \tau: \mathrm{m} \in \operatorname{dom}(\mathcal{G} c)$

early check

$\forall c \in \tau: \operatorname{dom}(\mathcal{G} c \mathrm{~m})=\{0, \ldots, n\}$

early check

$\forall c \in \tau: \tau_{i} \subseteq \mathcal{G} c \mathrm{~m} i$

subtype check

$\mathrm{E} . \mathrm{m}\left(\mathrm{E}_{1} \ldots \mathrm{E}_{n}\right):: \bigcup_{c \in \tau} \mathcal{G} c \mathrm{~m} 0$

Now, a program is statically correct when all method bodies have a typing in the corresponding environments.

Note that really only two kinds of checks are performed:

- early checks, which require the existence of certain declared names.

- subtype checks, which require inclusions between certain types.

With this definition of static correctness, it should be obvious that variables of type $\mathrm{T}$ can only contain objects of type $\mathrm{T}$; that if an expression is evaluated to a non-nil result, then the class of that result is contained in the type of the expression; and that we can guarantee that any message is sent to either nil 
or an instance of a class which implements a method for that message. Note that we ignore keeping track of nil-values; this can be treated separately by flow analysis.

A formal proof of these claims should be based on a dynamic semantics of the example language $[47,31]$. We will not go into the details in this paper, however, but move on to define a convenient representation of classes.

\subsection{Tree Representations of Classes}

We shall work with a slightly abstracted form of classes, which will allow us to give a formal treatment of their relationships.

The mathematical framework is a kind of labeled trees, which we shall call L-trees.

Definition 3.1: Let $\Sigma$ be a finite alphabet. An $L$-tree over $\Sigma$ is an ordered, node-labeled, regular tree. We recall that a tree is regular when it has only finitely many different subtrees [20]. The labels are finite strings over $\Sigma \cup$ $\{\bullet\}$, where it is assumed that $\bullet \notin \Sigma$. The empty label is denoted by $\Omega$. We shall refer to the special symbol $\bullet$ as a gap. It is further required that any node in an L-tree has exactly one subtree for each gap in its label.

We have some notation associated with such trees.

Definition 3.2: Let $T$ be an L-tree. A tree address is a sequence of integers denoting a path from the root to a node; each integer denotes the number of a subtree in the given order. We write $\alpha \in T$ when $\alpha$ is a valid tree address in $T$. The empty tree address is denoted by $\lambda$. If $\alpha \in T$ then $T[\alpha]$ denotes the label with address $\alpha$ in $T$, and $T \downarrow \alpha$ denotes the subtree of $T$ whose root has address $\alpha$. Note that $T \downarrow \lambda=T$.

We can now separate a class from its surrounding program and represent it as an L-tree. Intuitively, the tree is a possibly infinite unfolding of the source code of the class.

The labels will be source code with gaps in place of occurrences of class names. Recall that a class name may occur before new, after instanceOf, and inside type expressions. We shall also replace occurrences of selfClass with gaps. 
The root label of a class representation is the gapped source code of the class. Let the classes in the program be $C_{1}, \ldots, C_{n}$ and the corresponding root labels be $L_{1}, \ldots, L_{n}$. The trees $\operatorname{TREE}\left(\mathrm{C}_{1}\right) \ldots, \operatorname{TREE}\left(\mathrm{C}_{n}\right)$ are defined through the following regular equation system:

$$
\begin{aligned}
\operatorname{TREe}\left(\mathrm{C}_{1}\right) & =L_{1}\left(\bar{X}_{1}\right) \\
& \vdots \\
\operatorname{TREE}\left(\mathrm{C}_{n}\right) & =L_{n}\left(\bar{X}_{n}\right)
\end{aligned}
$$

Each $\bar{X}_{i}$ is a list of subtrees, which is obtained as follows. Every subtree corresponds to a gap in the label $L_{i}$. If the gap replaced the class $C_{j}$, then the subtree is $\operatorname{TREE}\left(C_{j}\right)$; if the gap replaced selfClass, then the subtree is $\operatorname{TREE}\left(\mathrm{C}_{i}\right)$. It is well-known that such an equation system has a unique solution [19], which clearly is an L-tree. If any part of a class is recursive, then the tree will be infinite.

Quite often, recursive types are represented as regular trees with nodes labeled by type constructors [2]. This is in fact what we have done, with the proviso that we consider every class as a user-defined type constructorrather than as a user-defined type.

We have now abstracted away from the class names, which obviously cannot be uniquely recovered. We can, however, transform a tree $T$ back into an equivalent program, PROG $(T)$, by selecting a class name for every different subtree in $T$ and reconstructing the syntax. We then have the property that $T \in \mathcal{U}$ if and only if $\operatorname{PROG}(T)$ is statically correct. Note that the structural equivalence on classes simply says that two classes are equivalent if their trees are equal.

It might be considered that the trees are not sufficiently abstract to deserve attention. For instance, we distinguish the order in which methods are implemented, and type expressions are interpreted as sequences rather than sets. Some tidying up is certainly possible: methods could be ordered in a canonical way, and type expressions could be normalized. For the purposes of this paper, however, such improvements are not really necessary. We shall mainly look at classes obtained as modifications of other classes, in which particular arbitrary choices are always carried along. The behavior of a class, for example an element of a Scott-domain, is clearly not a feasible representation-it is too abstract. 
We can now define a universe of classes as follows:

$$
\mathcal{U}=\{\operatorname{TREE}(\mathrm{C}) \mid \mathrm{C} \text { is a class in some statically correct program }\}
$$

This is a mathematical object on which we later shall observe an interesting structure.

\subsection{Examples}

We now provide some short examples of the above definitions. Consider

\begin{tabular}{|l|} 
class Object \\
end Object \\
class $A$ \\
$\quad$ var $x:$ Object \\
end $A$ \\
class $B$ \\
var $x:$ Object \\
method set(y:Object) returns selfClass \\
$\quad x:=y ;$ self \\
end $B$
\end{tabular}

Figure 4: Example classes

the classes A and B in figure 4. Let us first consider what the corresponding trees look like. We shall use the abbreviations

$$
\begin{aligned}
& L_{\mathrm{A}}=\operatorname{var} \mathrm{x}: \bullet \\
& L_{\mathrm{B}}=\operatorname{var} \mathrm{x}: \bullet \operatorname{method} \operatorname{set}(\mathrm{y}: \bullet) \text { returns } \bullet \mathrm{x}:=\mathrm{y} ; \text { self }
\end{aligned}
$$

The equation system for the trees is now

$$
\begin{aligned}
& \operatorname{TREE}(\text { Object })=\Omega() \\
& \operatorname{TREE}(\mathrm{A})=L_{\mathrm{A}}(\operatorname{TREE}(\text { Object })) \\
& \operatorname{TREE}(\mathrm{B})=L_{\mathrm{B}}(\operatorname{TREE}(\text { Object }), \operatorname{TREE}(\text { Object }), \operatorname{TREE}(\mathrm{B}))
\end{aligned}
$$


The corresponding trees are pictured in figure 5 . We can finally observe that

$$
\begin{aligned}
& \operatorname{TrEe}(\mathrm{B})[3,3,2]=\Omega \\
& \operatorname{TrEe}(\mathrm{B})[3,3,3]=L_{\mathrm{B}}
\end{aligned}
$$

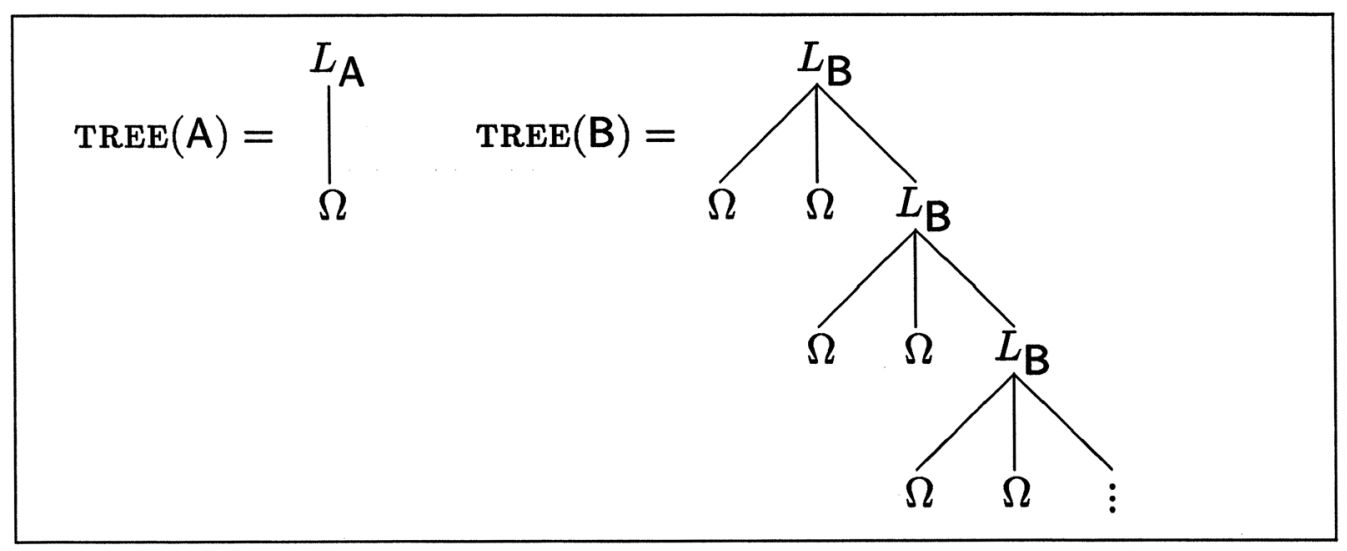

Figure 5: Example trees.

\section{Inheritance}

Inheritance is reuse of class definitions. It may significantly increase programmer productivity and decrease source code size. In this section we add inheritance to our example language, discuss its properties, and show that we can use the universe of class representations from the previous section to represent also the classes defined by inheritance.

\subsection{Syntax}

To introduce inheritance we extend the grammar in figure 3 as showed in figure 6 .

The class name following inherits is called the superclass of the class being defined; the latter is called a subcluss of the superclass. 


$$
\text { (Class) } \quad \begin{aligned}
\mathrm{C}::= & \text { class ClassId inherits ClassId } \\
& \text { var } \mathrm{D}_{1} \ldots \mathrm{D}_{k} \mathrm{M}_{1} \ldots \mathrm{M}_{n} \\
& \text { end ClassId }
\end{aligned}
$$

Figure 6: Syntax of Inheritance.

The subclass is a modification of the superclass: it may add variables and methods, and if a method name coincides with an existing one, then the new method definition overrides the old one. The body of a new method definition may refer to both the existing variables and the existing methods. It has been argued by Snyder [52] that much better encapsulation is achieved if only the existing methods can be referred; we ignore this consideration in this paper. For a denotational semantics of inheritance, see [17, 47, 31, 14].

\subsection{Properties}

Inheritance can be used in various ways, ranging from undisciplined codegrabbing to disciplined program structuring based on a hierarchical design method [21, 3, 13]. Common to all approaches is that the superclass is created before the subclass. We will henceforth use the terminology that the subclass is temporally dependent on its superclass.

A class can be a subclass of another class which itself is defined by inheritance. The chain of superclasses is always finite, however, because the program is finite. Also, the superclass chain will contain no cycles; we will say that it is temporally acyclic. Note that any non-empty class can be defined as a subclass of the empty class, which we will call Object. This is actually enforced in SMaLlTalK. In this situation, the inheritance hierarchy is a tree, otherwise it is a forest.

If $C$ is the superclass and $D$ is the subclass, then it is common to say that $D$ IS-A C [6, 54]. For example, if Student is a subclass of Person, then it seems reasonable to say that Student IS-A Person. It is convenient to let IS-A denote the transitive closure of this relation. The other possible relation between classes is HAS-A. If a class $C$ declares a variable of a type which contains the class D, then we will say that C HAS-A D. For example, if the Student declares a graduate variable of type Boolean, then Student HAS-A Boolean. 
We will also say that $C$ HAS-A $D$ if $C$ mentions $D$ in a parameter list or as an argument of new or instanceOf. Analogously with IS-A, we let HAS-A denote the transitive closure of this relation.

It is crucial not to confuse IS-A and HAS-A. If C HAS-A D and D HAS-A $C$, then we will say that $C$ and $D$ are mutually recursive. In comparison, it is impossible to have both C IS-A D and D IS-A C because that would be a temporal cycle. To make any sense, mutually recursive classes must be defined simultaneously by the programmer.

Together, IS-A and HAS-A impose a temporal order on the classes in a program. The intuition is that a class $D$ depends temporally on a class $C$ if $\mathrm{C}$ has to be created before $\mathrm{D}$. We will formalize this order in the following subsection, where we also show that a program that uses inheritance can be transformed into one which does not. Our reason for doing this is that when all classes are represented as elements of our universe of class representations, then it makes sense to analyze this universe in order to discover other relations between classes besides IS-A and HAS-A. Such relations will be independent both of class names and of the particular shorthands that can be used in program texts. So far, the only shorthand we have encountered is inheritance, but later on we will define another called class substitution. Pedersen [46] proposed the notion of generalization which is the inverse of inheritance.

The loss of an explicit class hierarchy may at first seem to cause severe problems, since some programming mechanisms depend on exactly this. In particular, we think about redefinition of method bodies in subclasses, and about the constructs super [25] and inner [33, 36]. However, these mechanisms depend primarily on the existence of multiple implementations of methods. This we can certainly handle, since a label contains a sequence of implementations of methods - several of which may have the same name. The dynamic behavior of a message send is to search the label from right to left, and to execute the first implementation of the method that is found. This gives the correct semantics of method redefinitions. The construct super can be viewed as a directive to search from the location of the present method implementation towards the left. Dually, the construct inner directs the search to go from the present location towards the right. This will nearly give the usual semantics, and is certainly in line with the explanation given in [5]. 
When expanding the inheritance shorthand it is necessary to be careful when encountering recursive occurrences of a class. A class $C$ is recursive if $C$ HASA C. If a class D inherits this class $C$, then after expansion, all occurrences of $C$ must have been transformed into $D$. The reason is that a variable of a type which contains $\mathrm{C}$ could be assigned to a variable which contains selfClass. Since selfClass always denotes the class it appears in, it will automatically denote D after the expansion. Hence, $C$ must be transformed into D to preserve static correctness. The complications get worse when considering mutually recursive classes; the algorithm in the following subsection gives a detailed solution to the general case.

While it is often the natural choice to transform all recursive occurrences during inheritance, one can certainly find program examples where the opposite choice is preferable. However, with our structural equivalence on classes recursive occurrences must be transformed in order to make the subclass statically correct. The problem could be solved by introducing opacity operators on classes, in line with [43]. In this paper we will not explore this aspect further.

\subsection{The Expansion Algorithm}

We now present the algorithm that expands a program using inheritance into an equivalent one that does not. The idea behind the algorithm is simple: it rewrites the program in the same fashion that a programmer would have to if the program should be implemented in a language that does not support inheritance.

In view of the preceding discussion, the program will be represented as a directed graph. There is one node for every class definition in the program. Each node has a label which is the gapped source code of the corresponding implementation. There will be two kinds of edges: IS-A (drawn with ordinary arrows) and HAS-A (drawn with dashed arrows). We have an IS-A edge from every subclass to its immediate superclass, and a HAS-A edge from every gap in the label to the node representing the missing class.

By a temporal dependency we shall understand a path between two nodes containing at least one IS-A edge. A temporal dependency from D to C means that $\mathrm{C}$ must be created before $\mathrm{D}$. 
From previous arguments we know that the graph has the following properties:

- every node has at most one outgoing IS-A edge.

- there are no temporal cycles; that is, no cycle in the graph can involve an IS-A edge.

The algorithm will transform this graph $(V, E)$ into a pair $(Q, \rho)$ where $Q$ is an equation system on regular trees, and $\rho$ is a map from nodes in the graph to variables in $Q$. The meaning is that a class with node w will denote the tree corresponding to the value of $\rho(v)$ in the unique solution of $Q$.

The algorithm proceeds iteratively, by processing the nodes of the graph in batches and removing them. A batch consists of all temporally independent nodes; that is, those nodes that have no outgoing path involving an IS-A edge. Since the graph is temporally acyclic, this is a non-empty set.

Such a batch has two special properties:

- all outgoing IS-A edges from nodes in the batch lead to earlier processed nodes.

- all outgoing HAS-A edges from nodes in the batch lead to earlier processed nodes or to other nodes in the batch.

The first follows easily from the nodes in the batch being temporally independent. The second can be seen from figure 7 where the general situation is sketched. Suppose we have a HAS-A edge from the node $v$ in the current batch to an unprocessed node $w$. Since $w$ is not temporally independent, then it has a temporal dependency to some other node $u$. By composing paths, we see that $v$ also has a temporal dependency to $u$. But this contradicts that $v$ belonged to the current batch of temporally independent nodes.

Thus we can expand the nodes in the current batch simultaneously. We maintain the invariant that the processed nodes are in the domain of $\rho$ and that $Q$ is a minimal equation system, i.e., no two variables denote the same tree. 


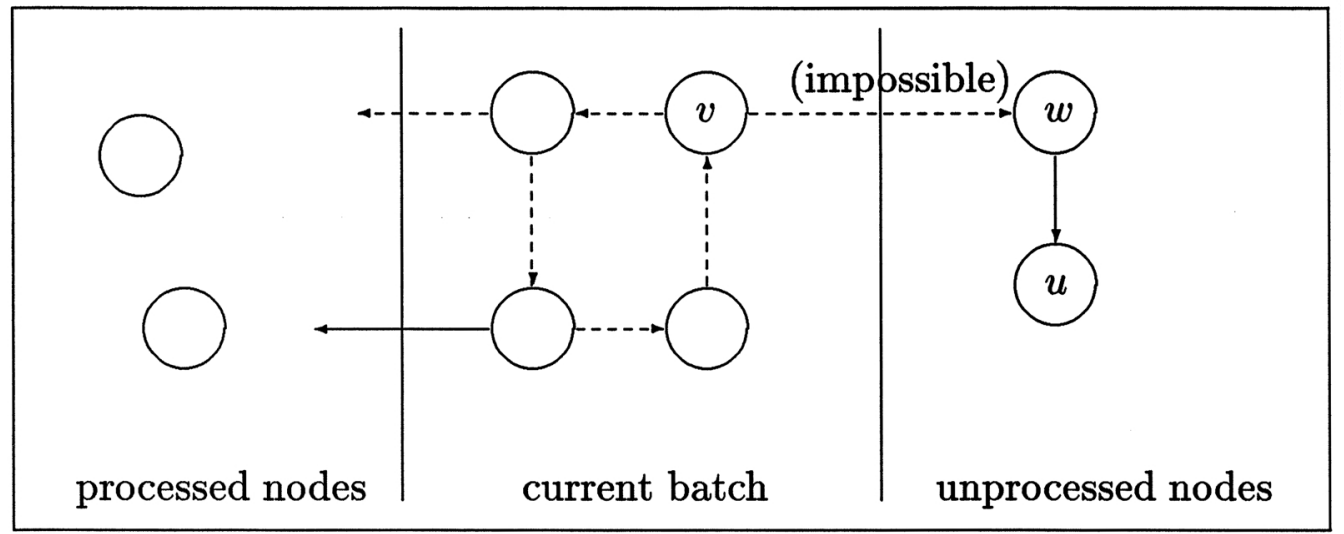

Figure 7: A stage in the algorithm.

Assume that the current batch consists of the nodes $\left\{b_{1}, b_{2}, \ldots, b_{n}\right\}$ with associated labels $L_{1}, L_{2}, \ldots, L_{n}$. To perform the expansion, we first introduce $n$ new variables $B_{1}, B_{2}, \ldots, B_{n}$. We also generate $n$ independent copies $Q_{1}, Q_{2}, \ldots, Q_{n}$ of the equation system $Q$; such a copy $Q_{i}$ is identical to the original, except that it has a fresh set of variables.

Looking at a particular node $b_{i}$ we have two cases.

- If $b_{i}$ has no superclass, then we simply introduce the equation

$$
B_{i}=L_{i}(\ldots)
$$

- If $b_{i}$ inherits the processed node $v$, then we look at the $Q_{i}$-version of the equation for $\rho(v)$, say

$$
X=L(\bar{Y})
$$

The equation is modified to

$$
\begin{aligned}
X & =L L_{i}(\bar{Y}, \ldots) \\
B_{i} & =X
\end{aligned}
$$

In both cases the extra arguments are determined as follows. If an argument corresponds to a HAS-A edge to a node $b_{j}$ in the current batch, then it is 
$B_{j}$. If it corresponds to a HAS-A edge to a processed node $w$, then it is the $Q_{i}$-version of $\rho(w)$.

Next, we update p such that $\rho\left(b_{i}\right)=B_{i}$, and finally we minimize $(Q, \rho)$. This may involve that several variables in $Q$ become identified, which is reflected in $\rho$ by several nodes being mapped to the common variable.

When the iteration terminates, then all nodes in the graph have been processed; hence, we have a tree associated with every class definition. In this general setting, we define $\operatorname{TREE}(\mathrm{C})$ to be the unique solution of $\rho(\mathrm{C})$ in $Q$. The entire algorithm is summarized in figure 8.

\begin{tabular}{|l|l|}
\hline $\begin{array}{l}\text { Input } \\
\text { Output }\end{array}$ & A program graph: $(V, E)$ \\
& The expansion: $(Q, \rho)$ \\
Algorithm: & $P, Q, \rho \leftarrow \emptyset, \emptyset, \emptyset$ \\
& do $P \neq V \rightarrow$ \\
& let $\left\{b_{1}, b_{2}, \ldots, b_{n}\right\}$ be the temporally \\
& independent nodes in $(V \backslash P, E)$ \\
& $Q \leftarrow Q \cup Q_{1} \cup Q_{2} \cup \ldots \cup Q_{n}$ \\
& for $i=1 . . n$ do \\
& add the equation for $b_{i}$ \\
& end \\
& update $\rho$ \\
& minimize $(Q, \rho)$ \\
& $P \leftarrow P \cup\left\{b_{1}, b_{2}, \ldots, b_{n}\right\}$ \\
end & \\
\hline
\end{tabular}

Figure 8: A summary of the expansion algorithm.

One may wonder how much larger a program can become after expansion. The crucial factor is the shape of the IS-A tree. If we have $n$ classes, then we must maximize the sum

$$
\sum_{i} \operatorname{depth}\left(\mathrm{C}_{i}\right)
$$

over all trees with nodes $\mathrm{C}_{1}, \ldots, \mathrm{C}_{n}$. The worst-case is a linear tree, which expands to size $O\left(n^{2}\right)$. A perfectly balanced tree only expands to size $O(n)$.

We may view the algorithm as giving a map ExPAND from classes with inheritance to classes without inheritance. If we consider a standard dynamic 
semantics of inheritance $[17,47,31,14]$, then we obtain a map SEM from classes - with and without inheritance - to denotations of meaning.

We now claim that the equation $\operatorname{SEM}(C)=\operatorname{SEM}(\operatorname{ExPAND}(C))$ holds for all classes $C$. We will not present a (rather voluminous) proof of this result, but move on to explore the structure of our universe of classes. First, however, we give an example.

\subsection{Example}

This subsection contains an example of how the expansion algorithm works.

\begin{tabular}{|ll|}
\hline class $U$ & class $W$ \\
var a: $U$ & var $c: V$ \\
end $U$ & end $W$ \\
& \\
class $V$ inherits $U$ & class $R$ inherits $V$ \\
var $b: W$ & var $d: W$ \\
end $V$ & end $R$ \\
\hline
\end{tabular}

Figure 9: Classes with inheritance.

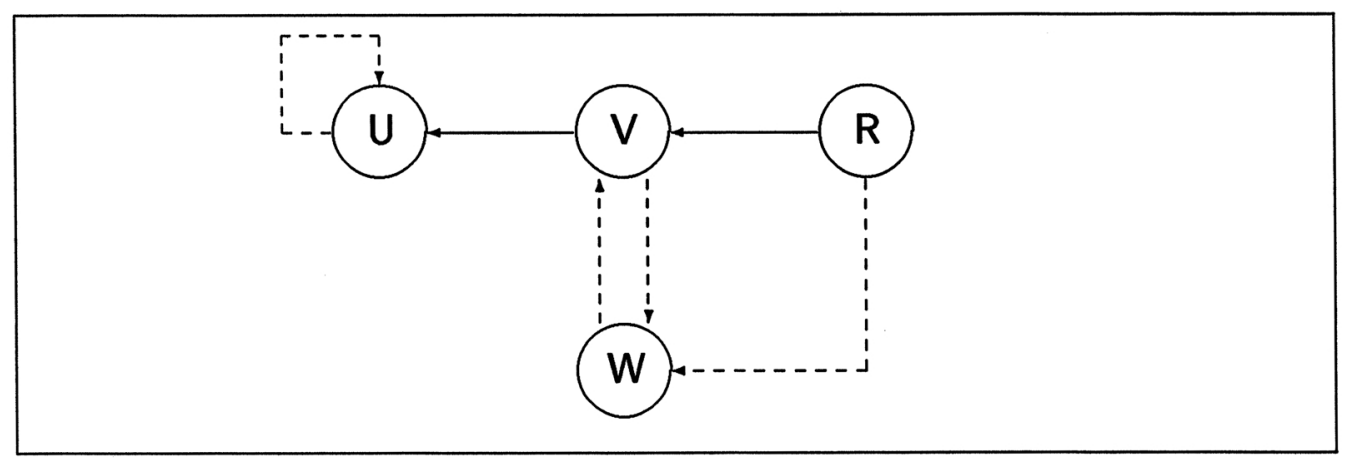

Figure 10: The program graph with inheritance.

Consider the classes in figure 9. We shall subject them to the expansion algorithm. The corresponding program graph is shown in figure 10. The reader is invited to try to expand the program by hand before reading on. We use the abbreviations 


$$
\begin{aligned}
& L_{\mathrm{U}}=\operatorname{var} \mathrm{a}: \bullet \\
& L_{\mathrm{V}}=\operatorname{var} \mathrm{b}: \bullet \\
& L_{\mathrm{W}}=\operatorname{var} \mathrm{c}: \bullet \\
& L_{\mathrm{R}}=\operatorname{var} \mathrm{d}: \bullet
\end{aligned}
$$

Initially, we start out with the following triple

\begin{tabular}{|l|l|l|}
\hline$P$ & $Q$ & $\rho$ \\
\hline \hline$\emptyset$ & $\emptyset$ & $\emptyset$ \\
\hline
\end{tabular}

The first batch of temporally independent nodes is $\{U\}$. Since no inheritance is involved, the first iteration gives us the triple

\begin{tabular}{|c|c|c|c|}
\hline$P$ & & $Q$ & $\rho$ \\
\hline בUU\} & $\begin{array}{l} \\
U \\
U\end{array}$ & $\begin{array}{ll}= & L_{\mathrm{U}}(U) \\
= & L_{\mathrm{U}}\left(U_{1}\right) \\
= & L_{\mathrm{U}}\left(U_{2}\right)\end{array}$ & $\overline{\langle(\mathrm{U}, U)\}}$ \\
\hline
\end{tabular}

\begin{tabular}{|c|c|c|}
\hline$P$ & $Q$ & $\rho$ \\
\hline \hline$\{\mathrm{U}\}$ & $U=L_{\mathrm{U}}(U)$ & $\{(\mathrm{U}, U)\}$ \\
\hline
\end{tabular}

The next batch is $\{\mathrm{V}, \mathrm{W}\}$. We first create the necessary copies of $Q$, yielding the triple

Next, we introduce the proper new equations

\begin{tabular}{|c|r|c|}
\hline$P$ & $Q$ & $\rho$ \\
\hline \hline$\{\mathrm{U}\}$ & $U=L_{\mathrm{U}}(U)$ & $\{(\mathrm{U}, U),(\mathrm{V}, V),(\mathrm{W}, W)\}$ \\
& $U_{1}=L_{\mathrm{U}} L_{\mathrm{V}}\left(U_{1}, W\right)$ & \\
& $V=U_{1}$ & \\
& $U_{2}=L_{\mathrm{U}}\left(U_{2}\right)$ & \\
& $W=L_{\mathrm{W}}(V)$ & \\
\hline
\end{tabular}

After minimizing $Q$ we have 


\begin{tabular}{|c|c|c|}
\hline$P$ & \multicolumn{1}{|c|}{$Q$} & $\rho$ \\
\hline \hline$\{\mathrm{U}, \mathrm{V}, \mathrm{W}\}$ & $U=L_{\mathrm{U}}(U)$ & $\{(\mathrm{U}, U),(\mathrm{V}, V),(\mathrm{W}, W)\}$ \\
& $V=L_{\mathrm{U}} L_{\mathrm{V}}(V, W)$ & \\
& $W=L_{\mathrm{W}}(V)$ & \\
\hline
\end{tabular}

The final batch is $\{\mathrm{R}\}$. We first create a copy of $Q$

\begin{tabular}{|c|c|c|c|}
\hline$P$ & & $Q$ & $\rho$ \\
\hline$\overline{q U}, \mathrm{~V}, \mathrm{~W}\}$ & $\begin{array}{c}t \\
V \\
W \\
U \\
V \\
W\end{array}$ & $\begin{array}{l}=L_{\mathrm{U}}(U) \\
=L_{\mathrm{U}} L_{\mathrm{V}}(V, W) \\
=L_{\mathrm{W}}(V) \\
=L_{\mathrm{U}}\left(U_{1}\right) \\
=L_{\mathrm{U}} L_{\mathrm{V}}\left(V_{1}, W_{1}\right) \\
=L_{\mathrm{W}}\left(V_{1}\right)\end{array}$ & 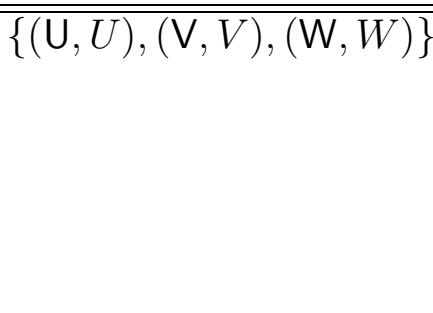 \\
\hline
\end{tabular}

Then we modify the equations

\begin{tabular}{|c|c|c|c|}
\hline$P$ & & $Q$ & $\rho$ \\
\hline$\{\mathrm{U}, \mathrm{V}, \mathrm{W}\}$ & $\begin{array}{r}t \\
V \\
W \\
U \\
V \\
I \\
W\end{array}$ & $\begin{array}{l}=L_{\mathrm{U}}(U) \\
=L_{\mathrm{U}} L_{\mathrm{V}}(V, W) \\
=L_{\mathrm{W}}(V) \\
=L_{\mathrm{U}}\left(U_{1}\right) \\
=L_{\mathrm{U}} L_{\mathrm{V}} L_{\mathrm{R}}\left(V_{1}, W_{1}, W_{1}\right) \\
=V_{1} \\
=L_{\mathrm{W}}\left(V_{1}\right)\end{array}$ & 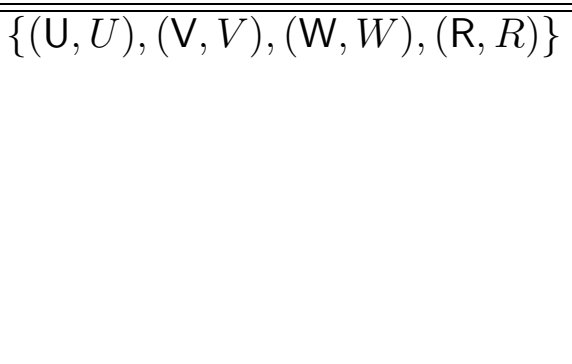 \\
\hline
\end{tabular}

After minimizing $Q$ we end up with

\begin{tabular}{|c|c|c|c|}
\hline$P$ & & $Q$ & $\rho$ \\
\hline$\{\mathrm{U}, \mathrm{V}, \mathrm{W}, \mathrm{R}\}$ & $\begin{array}{c}U \\
V \\
W \\
R \\
W_{1}\end{array}$ & $\begin{array}{l}=L_{\mathrm{U}}(U) \\
=L_{\mathrm{U}} L_{\mathrm{V}}(V, W) \\
=L_{\mathrm{W}}(V) \\
=V_{1} \\
=L_{\mathrm{W}}\left(V_{1}\right)\end{array}$ & 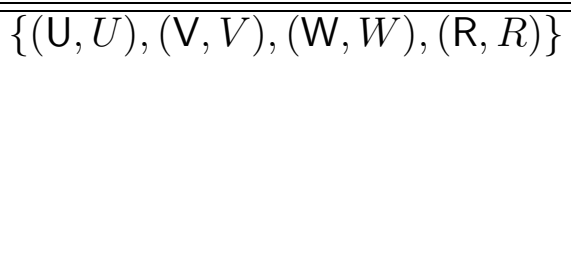 \\
\hline
\end{tabular}

The resulting classes are shown in figure 11. Notice the need for the class W1. The corresponding program graph is pictured in figure 12 . 


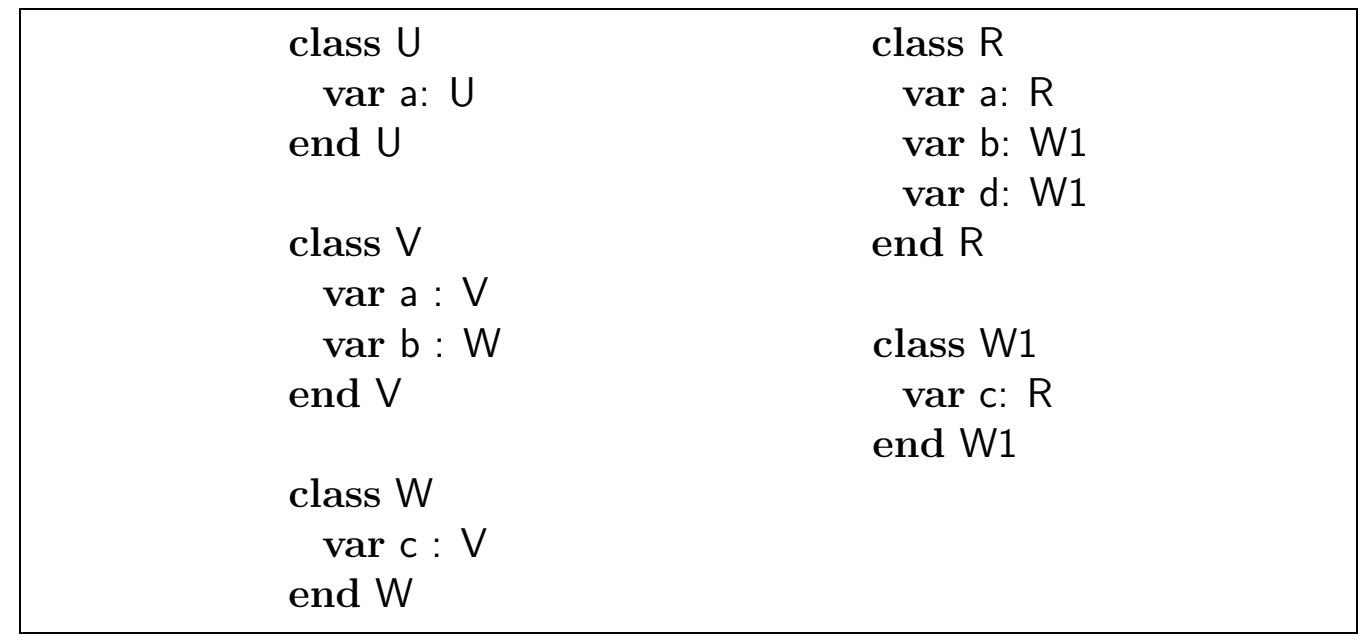

Figure 11: Classes without inheritance.

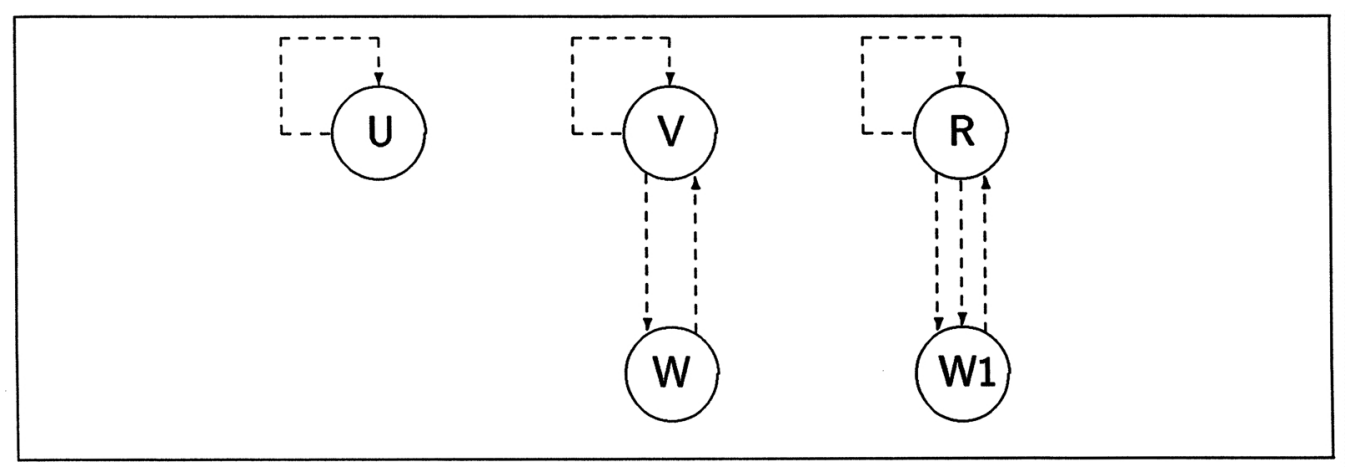

Figure 12: The program graph without inheritance.

\section{Generalized Subclassing}

The main purpose of providing an independent notion of classes is to define a generalized, structural notion of subclassing. This arises directly from the application on 24 of a partial order on general L-trees. 


\subsection{A Generalized Interpreter}

In the paper [45] we present a generalization of the standard SMALLTALK interpreter. The standard interpreter supports inheritance through a method lookup - a run-time search for implementations of methods. Our extended interpreter also does a run-time search for arguments to new and instanceOf operations. This clearly allows a more general form of code reuse, which we can express through a partial order $\triangleleft$ on classes. In [45] we give a precise description of the run-time environments of the extended interpreter, and we show the following property: if $T_{1} \triangleleft T_{2}$ holds, then any run-time implementation of $T_{1}$ can be extended to yield a runtime implementation of $T_{2}$. The code reuse that can be expressed through inheritance corresponds to a suborder of $\triangleleft$.

In this section we shall define the partial order $\triangleleft$ and show a number of its formal properties. In the following sections we shall develop a programming mechanism that is complementary to inheritance; as we shall see, their combination realizes all of $\triangleleft$.

\subsection{A Partial Order on Trees}

Intuitively, the relation $\triangleleft$ imposes three different constraints on subclasses. Each of these reflect that the subclass reuses the implementation of the superclass.

- the labels may only be extended: this simply means that the subclass can only extend the implementation and not modify existing parts. This also ensures that all early checks will remain satisfied.

- equal classes must remain equal: this ensures that all subtype checks will remain satisfied; hence, the code of the superclass can only be reused in a manner that preserves static correctness.

- the recursive structure must be preserved: this is essential for allowing the code to be reused since different code is generated for selfclass and other classes [45]. 
The partial order $\triangleleft$ is our generalized notion of subclassing, such that if $A$ is the superclass and $B$ is the subclass, then $A \triangleleft B$. It may seem strange that super is smaller than sub, but this is a common confusion of terminology. Clearly, the subclass has a larger implementation than the subclass; equally clearly, the superclass is more general than the subclass. We choose to retain the usual terminology, while employing the mathematically suggestive ordering $\triangleleft$.

To be able to define $\triangleleft$ formally, we introduce some auxiliary concepts. The first is a simple partial order on L-trees.

Definition 5.1: The usual prefix order on finite strings is written as $\leq$. The partial order $T_{1} \sqsubseteq T_{2}$ on L-trees over $\Sigma$ holds exactly when

- $\forall \alpha \in T_{1}: \alpha \in T_{2}$

- $\forall \alpha \in T_{1}: T_{1}[\alpha] \leq T_{2}[\alpha]$

Note that $\sqsubseteq$ is the node-wise extension of $\leq$.

The order $\sqsubseteq$ reflects that labels may only be extended. We next provide a simple, finite representation of an L-tree.

Proposition 5.2: Every L-tree $T$ can be represented by a finite, partial, deterministic automaton with labeled states, with language $\{\alpha \mid \alpha \in T\}$, and where a is accepted in a state labeled $T[\alpha]$.

Proof: The finitely many different subtrees all become accept states with the label of their root. The transitions of the automaton are determined by the fan-out from the corresponding root.

These automata provide finite representations of L-trees. The idea of representing a regular tree as an automaton is also exploited in [50, 51]. All later algorithms will in reality work on such automata.

Proposition 5.3: The partial order $\sqsubseteq$ is decidable.

Proof: The algorithm is a variation of the standard one for language inclu- 
sion on the corresponding automata.

The second auxiliary concept is the notion of a generator for an L-tree.

Definition 5.4: If $T$ is an L-tree over $\Sigma$, then its generator $\operatorname{GEN}(T)$ is another L-tree which is obtained from $T$ by replacing all maximal, proper occurrences of $T$ itself by a singleton tree with the special label $\diamond$; it is assumed that $\diamond$ is incomparable with all other labels in the $\leq$-ordering. We say that $T$ is recursive when $T \neq \operatorname{GEN}(T)$. Note that the generator itself may be an infinite tree, and that the original tree can readily be recovered from its generator.

The generator of a class makes explicit all the recursive occurrences of the class itself. For example, all occurrences of selfClass in its source code are replaced by $\diamond$, but also mutual recursion is captured.

We are now ready to define $\triangleleft$ using the order $\preceq$ which is a subset of $\sqsubseteq$.

Definition 5.5: The relation $T_{1} \preceq T_{2}$ on L-trees is the largest subset of $\sqsubseteq$ such that the following stability condition holds

- $\forall \alpha, \beta \in T_{1}: T_{1} \downarrow \alpha=T_{1} \downarrow \beta \Rightarrow T_{2} \downarrow \alpha=T_{2} \downarrow \beta$

The relation $T_{1} \triangleleft T_{2}$ on L-trees holds exactly when

- $\forall \alpha \in T_{1}: \operatorname{GEN}\left(T_{1} \downarrow \alpha\right) \preceq \operatorname{GEN}\left(T_{2} \downarrow \alpha\right)$

Note that if $T_{1} \triangleleft T_{2}$ then for any $\alpha \in T_{1}$ we also have $T_{1} \downarrow \alpha \triangleleft T_{2} \downarrow \alpha$.

Since $\preceq$ is a subset of $\sqsubseteq$, it reflects that labels may only be extended. Furthermore, the stability condition ensures that equal classes remain equal. The relation $\triangleleft$ is then defined so that the generators at all levels are in the $\preceq$ relation. This ensures that the recursive structure is preserved. 
Proposition 5.6: The relations $\preceq$ and $\triangleleft$ are decidable, partial orders.

Proof: Clearly, $\preceq$ is a partial order since stability is reflexive and transitive; also, $\triangleleft$ is a partial order because $\preceq$ is.

Since by proposition 5.3 we know that $\sqsubseteq$ is decidable, we must only show that stability is, too. On minimized automata, representing the trees $T_{1}$ and $T_{2}$, stability translates to the property that any two words $\alpha, \beta$ accepted in the same state by the $T_{1}$-automata must also be accepted in the same state by the $T_{2}$-automata. This property can be decided for general automata using a simple, linear-time dynamic programming algorithm.

To decide $\triangleleft$ we can rely on decidability of $\preceq$ and the fact that L-trees have only finitely many different subtrees, all of which can easily be constructed.

\subsection{Properties}

The subclassing order $\triangleleft$ has the same characteristic properties as inheritance: it has a least element, has finite intervals, does not allow temporal cycles, and preserves subtyping. In this subsection we prove these claims.

Proposition 5.7: The partial order $\triangleleft$ has a least element $\perp$. Proof: Clearly, $\perp$ is just the singleton tree with the label $\Omega$.

In a class hierarchy, $\perp$ corresponds to the empty class Object. To show that $\triangleleft$ has finite intervals, we need a notion of unfolding directed graphs.

Definition 5.8: Let $G$ be a directed, rooted graph containing a path from the root to each vertex. A particular unfolding of $G$, which we shall call $\operatorname{UNFOLD}(G)$, is obtained by the following variation of the standard depthfirst search algorithm [1] starting in the root. The modification is that if the current edge leads to a previously visited vertex in a different strongly connected component, then a fresh copy of that entire component is inserted in the graph. See for example figure 13 , where $\left(v_{1}, v_{3}\right)$ is the current edge. The graph $\operatorname{UnFOLD}(G)$ can be understood as a tree of possibly multiple copies of the strongly connected components of $G$. 


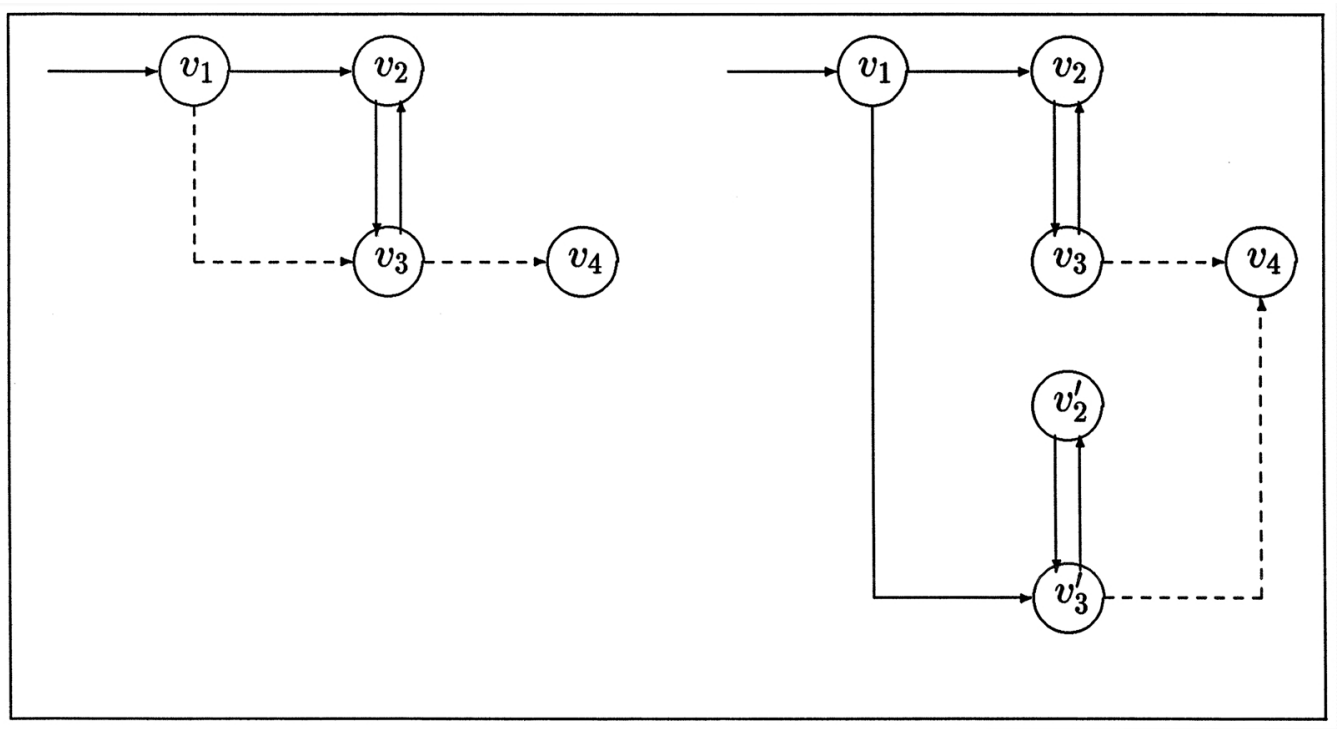

Figure 13: A step in the construction of $\operatorname{UNFOLD}(G)$.

Lemma 5.9: A depth-first traversal of $\operatorname{UNFOLD}(G)$ has the property that if the current edge leads to a previously visited vertex, then that vertex is on a cycle of already processed edges and the current edge.

Proof: Let $(v, w)$ be the current edge. If we have previously visited $w$, then, by construction of $\operatorname{UNFOLD}(G), v$ and $w$ are in the same strongly connected component. Because of the depth-first strategy, there is a path of already processed edges from $w$ to $v$. The result follows.

Proposition 5.10: For any L-tree $T_{2}$ the interval $\left\{T_{1} \mid T_{1} \triangleleft T_{2}\right\}$ is finite. Proof: For the purposes of this proof, we shall represent L-trees by their canonical automata. This is obtained by subjecting the minimal automaton to the unfolding described in definition 5.8. Clearly, this new automaton will have the same language and represent the same L-tree; in particular, the L-tree can be recovered from the automaton.

Now, assume that $T_{1} \triangleleft T_{2}$. Let $A_{1}$ and $A_{2}$ be their canonical automata. We shall construct a total function $\mathrm{h}$ from states of $A_{1}$ to states of $A_{2}$ with the following properties

- $h$ maps the initial state of $A_{1}$ to that of $A_{2}$ 
- if $x \stackrel{i}{\rightarrow} y$ is a transition in $A_{1}$, then $h(x) \stackrel{i}{\rightarrow} h(y)$ is a transition in $A_{2}$

- the label of $x$ is $\leq$ that of $h(x)$

- $h$ is injective

The construction works iteratively through a depth-first traversal of $A_{1}$. At any stage the current $h$ will satisfy all of the above properties, but it may be partial. We start with just the pair of initial states, which is clearly legal.

We proceed by examining the current unexplored depth-first $A_{1}$-transition $x \stackrel{i}{\rightarrow} y$ from a state $x$ in the domain of $h$. This is matched by an $A_{2}$-transition $h(x) \stackrel{i}{\rightarrow} z$, since the label of $x$ is $\leq$ than that of $h(x)$. The function $h$ is now extended to $h^{\prime}=h \cup\{y \mapsto z\}$. Only two necessary properties are not immediate: that $h^{\prime}$ is still a function, and that $h^{\prime}$ is still injective.

Assume that we have already seen $y$ before; we must assure that $z=h(y)$. Having seen $y$ before means, from lemma 5.9, that we have a cycle from $y$ to $y$. Now look at the generator of the subtree of $T_{1}$ that corresponds to $y$. The cycle that we have traversed is here a path from the root to a $\diamond$-label. In the $h(y)$-generator of $T_{2}$ the same path must also lead to a $\diamond$-label, since no other label can satisfy the $\sqsubseteq$-requirement. Hence, the path from $y$ to $y$ in $A_{1}$ translates to a path from $h(y)$ to $h(y)$ in $A_{2}$. It follows that $z=h(y)$.

Similarly, injectivity follows. If for some $x^{\prime}$ we have $z=h\left(x^{\prime}\right)$, then the cycle from $z$ to $z$ in $A_{2}$ must correspond to a cycle in $A_{1}$, from which it follows that $x=x^{\prime}$.

Since all states in a canonical automaton can be reached from the initial state, this construction will terminate with a total function.

To proceed, we observe that the existence of any injective function from states of $A_{1}$ to states of $A_{2}$ assures that there are no more states in $A_{1}$ than in $A_{2}$. Since any label in $A_{1}$ must be $\leq$ than some label in $A_{2}$, and we know that $\leq$ has finite intervals, then any $A_{1}$ must be built out of a bounded number of states and a finite set of labels. For simple combinatorial reasons, there can only be finitely many such automata.

Since different L-trees yield different canonical automata, the result follows. 
In particular, this result means that any class can only have finitely many superclasses.

Corollary 5.11: For any two L-trees $T_{1}, T_{2}$ we have that the closed interval $\left\{S \mid T_{1} \triangleleft S \triangleleft T_{2}\right\}$ is finite.

Proof: This is just a subset of the finite interval in proposition 5.10.

Next, we can show that our generalized notion of subclassing does not allow any temporal cycles. Since in our framework

$$
T_{1} \text { HAS-A } T_{2} \text { iff } \exists \alpha: T_{1} \downarrow \alpha=T_{2}
$$

and

$$
T_{1} \text { IS-A } T_{2} \text { iff } T_{2} \triangleleft T_{1} \wedge T_{2} \neq T_{1}
$$

then, to eliminate temporal cycles of the form $T$ HAS-A $S$ IS-A $T$, we must show that no tree can be strictly $\triangleleft$-less than one of its subtrees. Longer cycles are handled by transitivity and essentially the same argument.

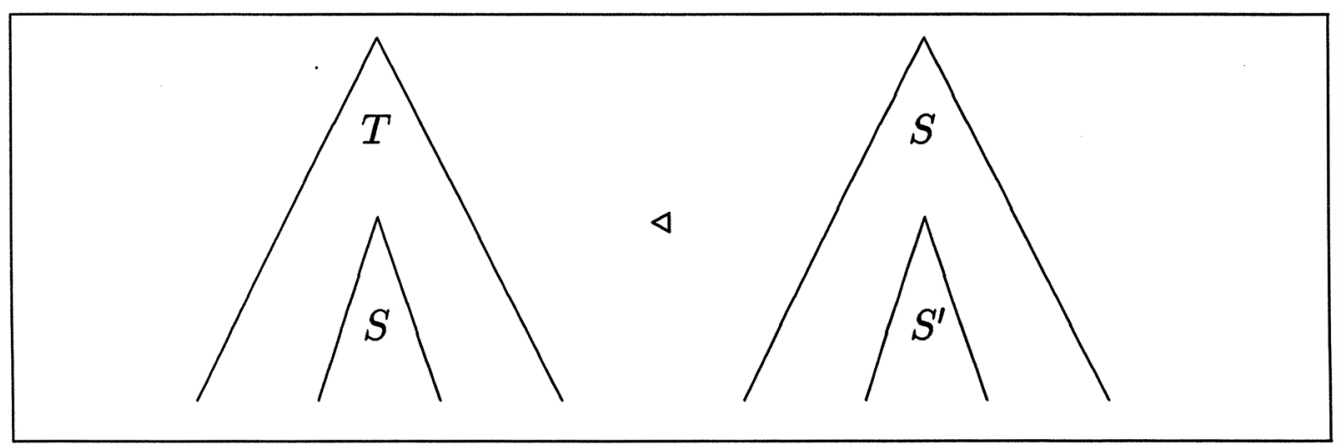

Figure 14: A temporal cycle.

Theorem 5.12: Let $T$ by an L-tree. If $T \triangleleft T \downarrow \alpha$ then $T=T \downarrow \alpha$. Proof: Assume that $S=T \downarrow \alpha, T \triangleleft S$, and $T \neq S$. Let $S^{\prime}=S \downarrow \alpha$. We must have $S \triangleleft S^{\prime}$, as illustrated in figure 14. If $S=S^{\prime}$ then the generator of $S$ has a $\diamond$-label in position $\alpha$. But then the generator of $T$ must also have 
a $\diamond$-label in position $\alpha$, which implies that $T=S$. Since we have assumed the opposite, we conclude that $S \neq S^{\prime}$. But then we can iterate the above construction and obtain a strictly $\triangleleft$-increasing chain

$$
T \triangleleft T \downarrow \alpha \triangleleft T \downarrow \alpha^{2} \triangleleft \downarrow \alpha^{3} \triangleleft \cdots \triangleleft T \downarrow \alpha^{i} \triangleleft \cdots
$$

In particular, this means that $T$ has infinitely many different subtrees, which contradicts its being an L-tree. The result follows.

A final property can be phrased as the slogan subclassing preserves subtyping. Intuitively, this means that subtype relationships will be preserved in subclasses.

Proposition 5.13: A type expression in a class $T$ consists of, say, $n$ classes located as the subtrees at addresses $\alpha_{1}, \alpha_{2}, \ldots, \alpha_{n}$; that is, the expression denotes the set

$$
A=\left\{T \downarrow \alpha_{1}, T \downarrow \alpha_{2}, \ldots, T \downarrow \alpha_{n}\right\}
$$

Suppose also we have another type expression denoting the set

$$
B=\left\{T \downarrow \beta_{1}, T \downarrow \beta_{2}, \ldots, T \downarrow \beta_{m}\right\}
$$

and that the inclusion $A \subseteq B$ holds. Let $S$ be any subclass of $T$. We then have two corresponding sets

$$
A^{\prime}=\left\{S \downarrow \alpha_{1}, S \downarrow \alpha_{2}, \ldots, S \downarrow \alpha_{n}\right\} B^{\prime}=\left\{S \downarrow \beta_{1}, S \downarrow \beta_{2}, \ldots, S \downarrow \beta_{m}\right\}
$$

and we are guaranteed that the inclusion $A^{\prime} \subseteq B^{\prime}$ will also hold.

Proof: This follows immediately from the stability requirement on $\triangleleft$.

\subsection{Examples}

To illustrate the concepts introduced in this section we continue the example from subsection 3.4.

The automata cokresponding to the classes $A$ and $B$ are shown in figure 15 . We can also observe that TREE(A) $\sqsubseteq \operatorname{TREE}(B)$. 


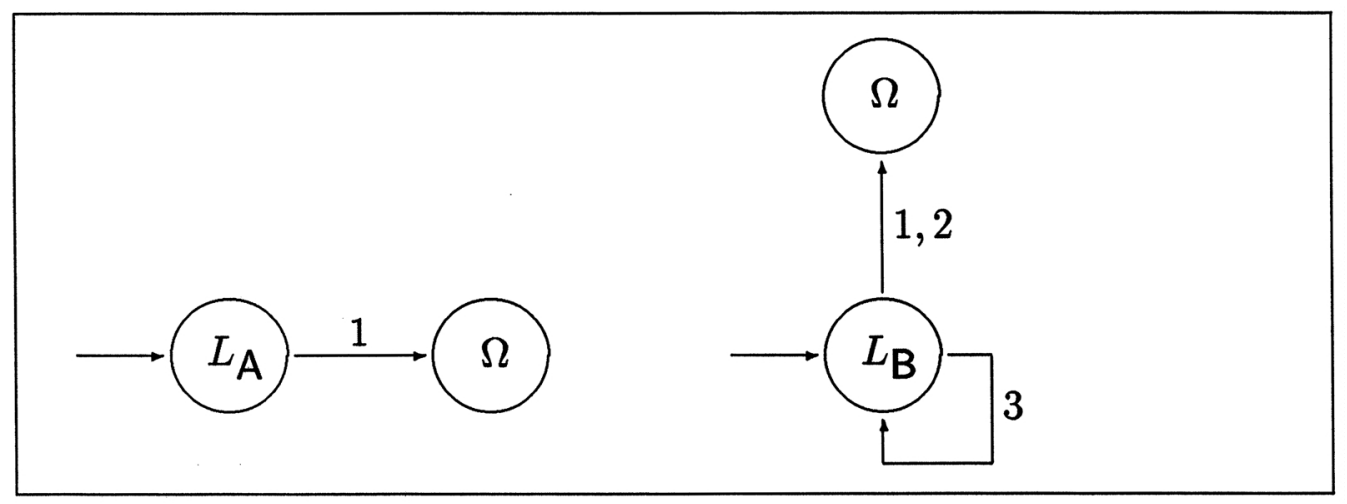

Figure 15: Example automata.

\begin{tabular}{|l|}
\hline class $C$ \\
var $h: B$ \\
var t: selfClass \\
end $C$ \\
class $C$ inherits $C$ \\
var z: Object \\
end D \\
\hline
\end{tabular}

Figure 16: Example classes.

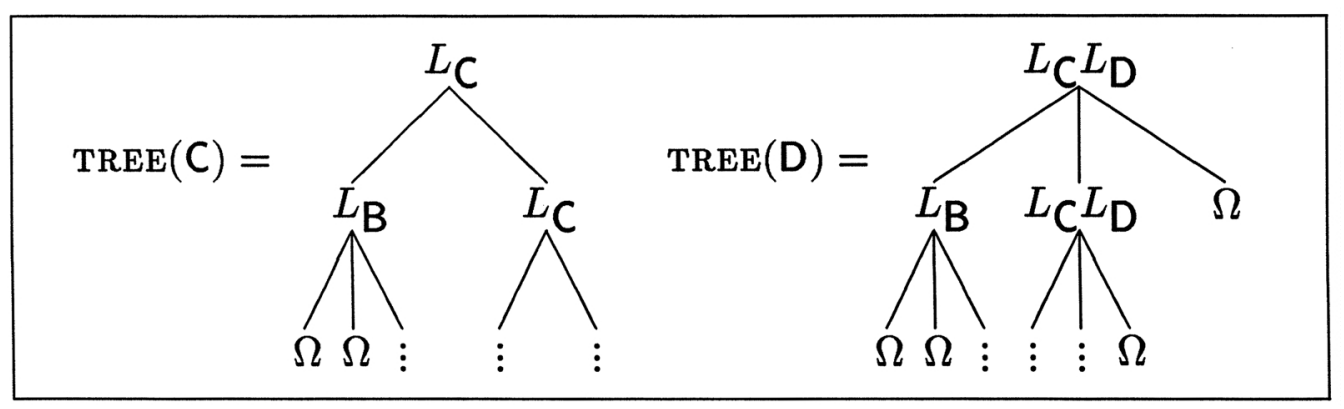

Figure 17: Example trees.

We next program two new classes $C$ and $D$ shown in figure 16. As before, we 


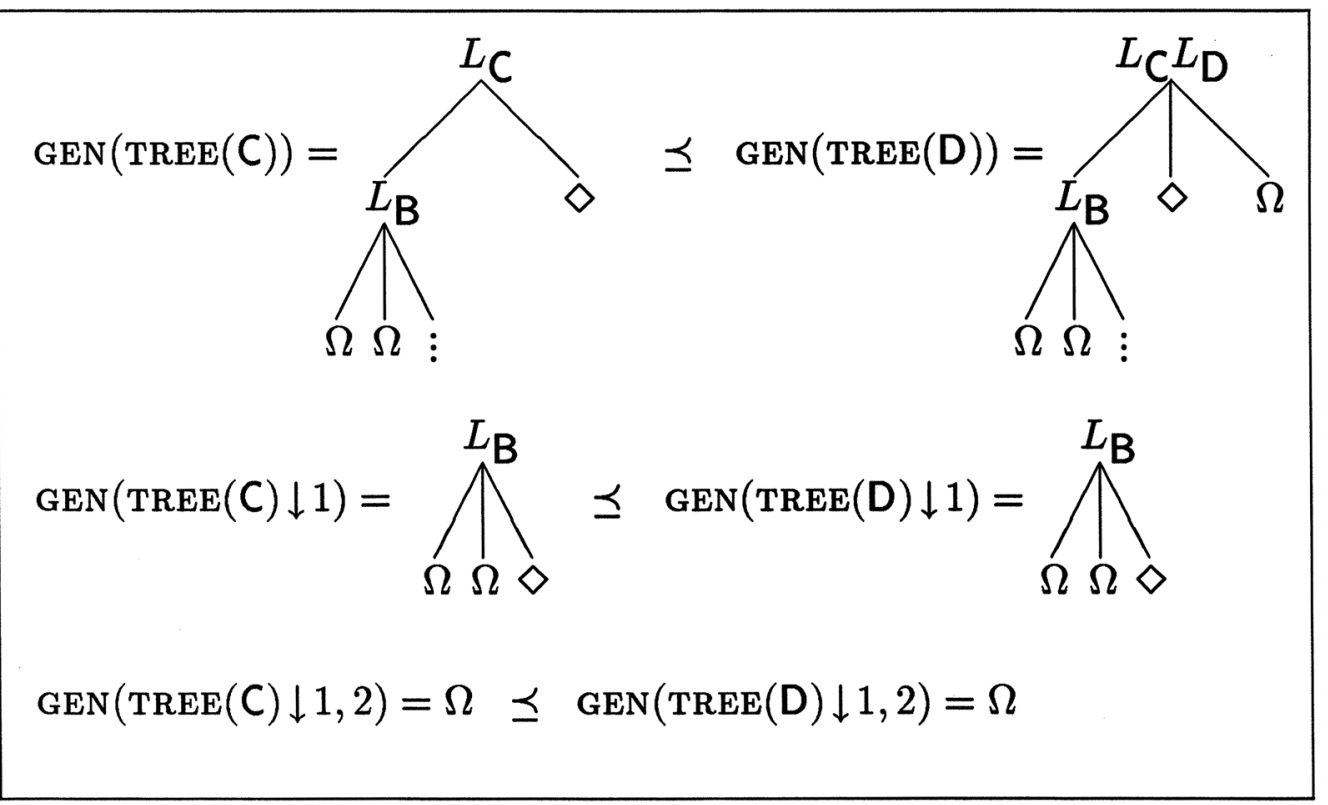

Figure 18: Relating generators.

define

$$
\begin{aligned}
& L_{\mathrm{C}}=\operatorname{var} \mathrm{h}: \bullet \operatorname{var} \mathrm{t}: \bullet \\
& L_{\mathrm{D}}=\operatorname{var} \mathrm{z}:
\end{aligned}
$$

The corresponding trees, shown in figure 17 , are defined by the equations

$$
\begin{aligned}
\operatorname{TreE}(\mathrm{C}) & =L_{\mathrm{C}}(\operatorname{TreE}(\mathrm{B}), \operatorname{TreE}(\mathrm{C})) \\
\operatorname{TreE}(\mathrm{D}) & =L_{\mathrm{C}}{ }^{L} \mathrm{CD}(\operatorname{TreE}(B), \operatorname{TreE}(D), \operatorname{TreE}(\text { Object }))
\end{aligned}
$$

Let us show that $\operatorname{TREE}(\mathrm{C}) \triangleleft \operatorname{TREE}(\mathrm{D})$. We have three different situations where a generator in TREE(C) must be $\preceq$ than a similar generator in TREE(D). Examples of all three situations are shown in figure 18. A tree that is $\preceq$ than TREE(D) but not $\triangleleft$ is shown in figure 19; it is not recursive, while TREE(D) is. 


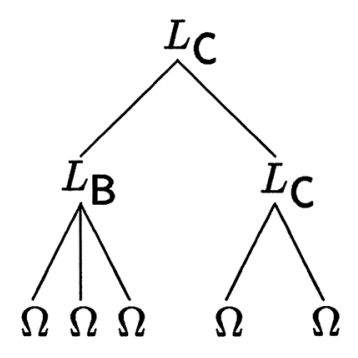

Figure 19: A non-recursive tree.

\section{The Orthogonality Result}

Inheritance is a programming mechanism which can realize only part of $\triangleleft$; more precisely, it captures a suborder.

\subsection{Two Suborders}

Definition 6.1: The partial order $T_{1} \triangleleft T_{2}$ holds exactly when

- $T_{1} \triangleleft T_{2} \wedge \forall \alpha \in T_{1}: T_{1} \downarrow \alpha \neq T_{1} \Rightarrow T_{1}[\alpha]=T_{2}[\alpha]$

This states that only the root label-and its recursive occurrences - may change.

The $\triangleleft_{I}$-part of $\triangleleft i s$ just inheritance.

Proposition 6.2: If $C_{1}$ is inherited by $C_{2}$ in any program, then $\operatorname{TREE}\left(C_{1}\right) \triangleleft_{I}$ $\operatorname{TREE}\left(\mathrm{C}_{2}\right)$. Conversely, if $T_{1} \triangleleft_{I} T_{2}$ then any $\mathrm{C}_{1}$ such that $\operatorname{TREE}\left(\mathrm{C}_{1}\right)=T_{1}$ can be modified by inheritance to yield a $\left(C_{2}\right)$ such that $\operatorname{TREE}\left(C_{2}\right)=T_{2}$.

Proof: Consider the isomorphism between L-trees and minimal equation systems. It is quite easy to see that $\triangleleft_{I}$ in this framework exactly captures the constructions performed by the expansion algorithm. 
The remaining part of $\triangleleft$ can be characterized in a satisfying manner: as an orthogonal complement of $\triangleleft_{I}$, in the following sense.

Definition 6.3: Let $P$ be a partial order on a set $S$. We use the notation $\triangle(S)$ for the diagonal $\{(s, s) \mid s \in S\}$, and the notation $A^{*}$ for the reflexive, transitive closure of a relation $A$. We write $Q \perp_{P} R$, if $Q$ and $R$ are partial orders such that $Q \cap R=\triangle(S)$ and $(Q \cup R)^{*}=P$. We call $Q, R$ an orthogonal basis for $P$ when

- $Q \perp_{P} R$

- $Q^{\prime} \perp_{P} R \Rightarrow Q \subseteq Q^{\prime}$

- $Q \perp_{P} R^{\prime} \Rightarrow R \subseteq R^{\prime}$

This generalizes the notion of basis in [26].

For example, if $\left(S_{1}, \leq_{1}\right)$ and $\left(S_{2}, \leq_{2}\right)$ are partial orders, then $\leq_{1} \times \triangle\left(S_{2}\right)$ and $\triangle\left(S_{1}\right) \times \leq_{2}$ form an orthogonal basis for $\leq_{1} \times \leq_{2}$.

The remaining part of $\triangleleft$ can be captured by the following suborder.

Definition 6.4: The partial order $T_{1} \triangleleft_{S} T_{2}$ holds exactly when

- $T_{1} \triangleleft T_{2} \wedge T_{1}[\lambda]=T_{2}[\lambda]$

This states that the root label must remain unchanged.

\subsection{Orthogonality}

We can now show that $\triangleleft_{I}, \triangleleft_{S}$ is an orthogonal basis for $\triangleleft$. This result is important, since it allows us to simply search for a programming mechanism that relates to $\triangleleft_{S}$ in the same fashion that inheritance relates to $\triangleleft_{I}$; the less appealing choice was to find a mechanism directly for the awkward set difference of $\triangleleft$ and $\triangleleft_{I}$. Furthermore, when we have such a $\triangleleft_{S}$-mechanism, then it is orthogonal to inheritance in a formal sense. The next chapter discloses 
that as yields a form of genericity. We have thus shown that inheritance and genericity are independent, orthogonal components of generalized subclassing.

To prove the result we need a series of lemmas.

Lemma 6.5: The relations $\triangleleft_{I}, \triangleleft_{S}$ as are both partial orders, and $\triangleleft_{I} \cap \triangleleft_{S}=$ $\triangle(\mathcal{U})$.

Proof: Clearly, $\triangleleft_{S}$ is a partial order. The extra condition on $T_{1} \triangleleft_{I} T_{2}$ simply means that for every $\alpha \in \operatorname{GEN}\left(T_{1}\right)$ we have $\operatorname{GEN}\left(T_{1}\right)[\alpha]=\operatorname{GEN}\left(T_{2}\right)[\alpha]$, except for the root labels which are $\leq$-related. Hence, $\triangleleft_{I}$ is a partial order. If also $T_{1} \triangleleft_{S} T_{2}$ then all labels must be equal, so the generators, and the trees, are equal.

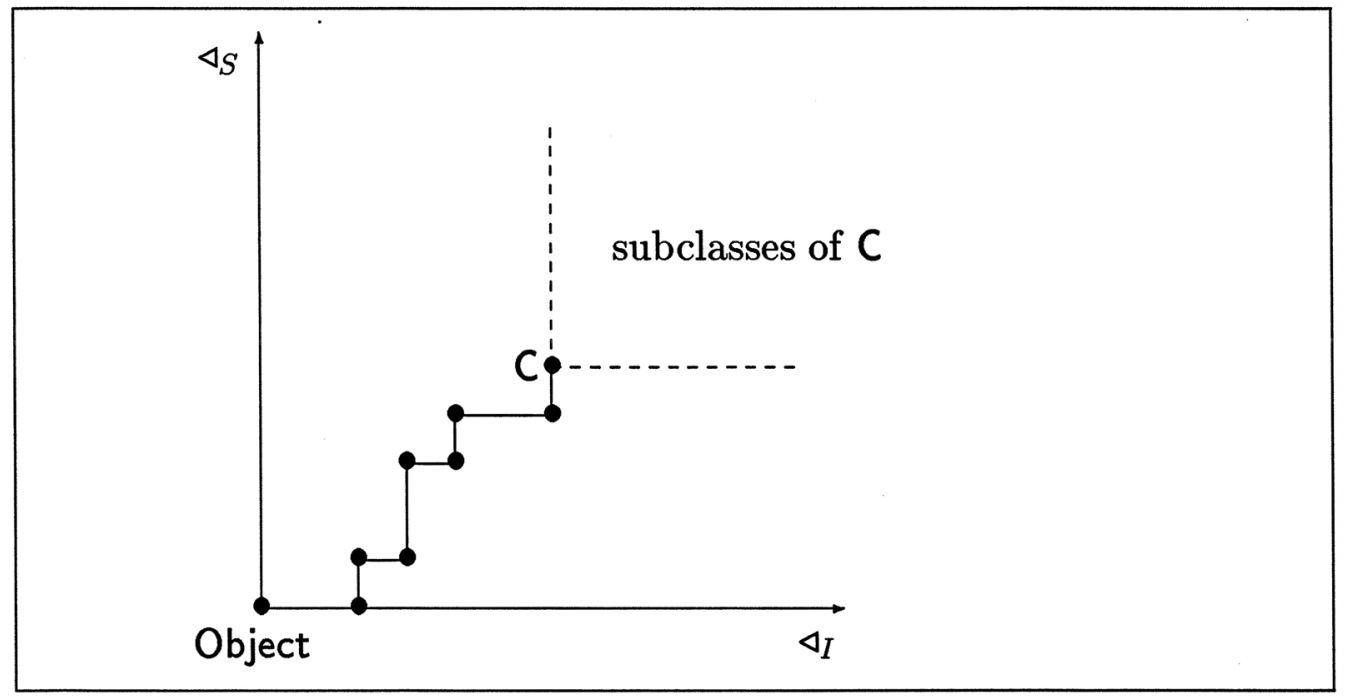

Figure 20: Orthogonal suborders.

Lemma 6.6: Whenever $T_{1} \triangleleft T_{2}$ then there is a unique $A \in \mathcal{U}$ such that $T_{1} \triangleleft_{S} A \triangleleft_{S} T_{2}$.

Proof: Suppose $T_{1} \triangleleft T_{2}$. Then $\operatorname{GEN}\left(T_{1}\right) \preceq \operatorname{GEN}\left(T_{2}\right)$. Let $L_{1}$ be the root label of $\operatorname{GEN}\left(T_{1}\right)$. Then the root label of $\operatorname{GEN}\left(T_{2}\right)$ must look like $L_{1} L 2$. Let $\operatorname{GEN}(A)$ be obtained from $\operatorname{GEN}\left(T_{2}\right)$ by removing the $L_{2}$-part of the root label and the subtrees that correspond to its gaps. Since subtrees with the same address in $\triangleleft$-related trees also will be $\triangleleft$-related, it follows that $T_{1} \triangleleft A$. Since 
$T_{1}, T_{2} \in \mathcal{U}$, then clearly $A \in \mathcal{U}$. But since they both have root label $L_{1}$, we also have $T_{1} \triangleleft_{S} A$. It is trivially the case that $A \triangleleft_{I} T_{2}$, so we have shown that $T_{1} \triangleleft_{S} A \triangleleft_{I} T_{2}$.

For the uniqueness of $A$, suppose we also have $T_{1} \triangleleft_{S} B \triangleleft_{I} T_{2}$. Then for every $\alpha \in \operatorname{GEN}\left(T_{2}\right)$ we have $\operatorname{GEN}\left(T_{2}\right)[\alpha]=\operatorname{GEN}(A)[\alpha]=\operatorname{GEN}(B)[\alpha]$, except for the root labels; but we also have $T_{1}[\lambda]=A[\lambda]=B[\lambda]$, so $A=B$.

Lemma 6.7: $\left(\triangleleft_{I} \cup \triangleleft_{S}\right)^{*}=\triangleleft$

Proof: Immediate from lemma 6.6.

Lemma 6.8: No partial order $\triangleleft_{M}$ which is a proper subset of $\triangleleft_{S}$ satisfies $\left(\triangleleft_{I} \cup \triangleleft_{M}\right)^{*}=\triangleleft$. Also, no partial order $\triangleleft_{N}$ which is a proper subset of $\triangleleft_{I}$ satisfies $\left(\triangleleft_{N} \cup \triangleleft_{S}\right)^{*}=\triangleleft$.

Proof: suppose we have such a $\triangleleft_{M}$. Choose $(x, y) \in \triangleleft_{S} \backslash \triangleleft_{M}$. Then $x[\lambda]=y[\lambda]$, so no $\triangleleft_{I} \triangle(\mathcal{U})$ steps can take place on a path from $x$ to $y$. Hence, $(x, y) \in \triangleleft_{M}^{*}=\triangleleft_{M}$ which is a contradiction. The other half of the result is proved similarly.

Lemma 6.9: Let $P$ be a partial order, where all closed intervals are finite. Whenever $P_{1}, P_{2} \subseteq P$ and $P_{1}^{*}=P_{2}^{*}=P$, then $\left(P_{1} \cap P_{2}\right)^{*}=P$.

Proof: Clearly, $\left(P_{1} \cap P_{2}\right)^{*} \subseteq P$. For the opposite inclusion, suppose $(x, y) \in$ $P$. The proof is by induction in the size of the open interval over $P$ from $x$ to $y$. If the interval is empty, then either $(x, y) \in\left(P_{1} \cap P_{2}\right)^{0}$, or $(x, y) \in\left(P_{1} \cap P_{2}\right)$. Now, suppose the interval contains $n+1$ elements. Choose $z$ in it. Then both the open interval from $x$ to $z$ and that from $z$ to $y$ contain at most $n$ elements. Hence, by the induction hypothesis, $(x, z),(z, y) \in\left(P_{1} \cap P_{2}\right)^{*}$. By transitivity of $\left(P_{1} \cap P_{2}\right)^{*}$ we conclude $(x, y) \in\left(P_{1} \cap P_{2}\right)^{*}$.

Lemma 6.10: If $\triangleleft_{M} \perp_{\triangleleft} \triangleleft_{S}$ as then $\triangleleft_{I} \subseteq \triangleleft_{M}$. Also, if $\triangleleft_{I} \perp_{\triangleleft} \triangleleft_{N}$ then as $\triangleleft_{S} \subseteq \triangleleft_{N}$. Proof: Suppose $\triangleleft_{M} \perp_{\triangleleft} \triangleleft_{S}$ as. By corollary 5.11, all closed $\triangleleft-$ intervals of $\mathcal{U}$ are finite, so by lemmas 6.7 and $6.9, \triangleleft=\left(\left(\triangleleft_{I} \cup \triangleleft_{S}\right)^{*}\right) \cap\left(\triangleleft_{M} \cup\right.$ $\left.\left.\triangleleft_{S}\right)\right)^{*}=\left(\left(\triangleleft_{I} \cap \triangleleft_{M}\right) \cup \triangleleft_{S}\right)^{*}$. By lemma $6.8, \triangleleft_{I} \cap \triangleleft_{M}$ cannot be a proper subset of $\triangleleft_{I}$. Hence, $\triangleleft_{I} \cap \triangleleft_{M}=\triangleleft_{I}$, so $\triangleleft_{I} \subseteq \triangleleft_{M}$ The other half of the lemma is proved similarly. 
Theorem 6.11: $\triangleleft_{I}, \triangleleft_{S}$ is an orthogonal basis for $\triangleleft$. Proof: Combine lemmas 6.5, 6.7, and 6.10.

The significance of this result is that a programming mechanism realizing $\triangleleft_{S}$, together with inheritance which realizes $\triangleleft_{I}$, allow the programming of all subclasses. Furthermore, two such programming mechanisms would be completely non-redundant; neither could emulate the other. The situation is illustrated in figure 20, which shows how all subclasses can be reached in axis-parallel moves.

\section{Class Substitution}

The suborder $\triangleleft_{S}$ requires that the root label cannot change. In terms of classes, this means that only type information may change, and not the implementation itself. This is precisely what intuitively characterizes genericity. This section introduces a programming mechanism that corresponds to $\triangleleft_{S}$. It is called class substitution and implements a general form of genericity.

\subsection{Syntax}

The syntax for class substitution is as follows. If $\mathrm{C}, \mathrm{A}_{i}$, and $\mathrm{B}_{i}$ are classes, then

$$
\mathrm{C}\left[\mathrm{A}_{1}, \ldots, \mathrm{A}_{n} \leftarrow \mathrm{B}_{1}, \ldots, \mathrm{B}_{n}\right]
$$

is a class substitution which specifies a class $D$ such that $C \triangleleft_{S} D$ and all occurrences of $A_{i}$ are substituted by $B_{i}$. If such a class does not exist, then the specification is statically incorrect.

\subsection{Semantics}

In this section we define precisely what class substitution means, and we show that it exactly realizes $\triangleleft_{S}$.

The algorithm to expand a substitution specification is summarized in figure 21. Intuitively, the relation $M$ collects all the individual substitutions that must be performed. The requirement that $M$ is $\triangleleft$-increasing is necessary in 


\section{Input A substitution specification: $C\left[A_{1}, \ldots, A_{n} \leftarrow B_{1}, \ldots, B_{n}\right]$ Output Either fail or a resulting class: D}

Algorithm: $M \leftarrow\left\{\left(\mathrm{A}_{i} \downarrow \alpha, \mathrm{B}_{i} \downarrow \alpha\right) \mid 1 \leq i \leq n, \alpha \in \mathrm{A}_{i}, \alpha \in \mathrm{B}_{i}\right\}$ if $M$ is not a $\triangleleft$-increasing, partial function then fail apply $M$ to the maximal subtrees of $\mathrm{C}$ in $\operatorname{dom}(M)$ yielding $D$

if not $\mathrm{D} \in \mathcal{U}$ and $\mathrm{C} \triangleleft_{S} \mathrm{D}$ then fail

Figure 21: Expanding substitutions.

order for $\mathrm{D}$ to be a subclass of $\mathrm{C}$. The requirement that $M$ is a function is necessary to avoid inconsistent substitutions. Note that the maximal subtrees of $\mathrm{C}$ that belong to the domain of $M$ is a uniquely determined set of disjoint subtrees. Note also that failed substitutions can be determined on compile-time.

Proposition 7.1: If $\mathrm{C} \triangleleft_{S} \mathrm{D}$ then

$$
\mathrm{D}=\mathrm{C}\left[\mathrm{A}_{1}, \ldots, \mathrm{A}_{n} \rightarrow \mathrm{B}_{1}, \ldots, \mathrm{B}_{n}\right]
$$

for some $\mathrm{A}_{i}, \mathrm{~B}_{i}$.

Proof: Clearly, $\mathrm{D}=\mathrm{C}[\mathrm{C} \leftarrow \mathrm{D}]$.

Hence, all $\triangleleft_{S}$-related subclasses can be expressed in this manner. Note though that the specification given in the proof of proposition 7.1 is rather useless for practical programming: it corresponds to writing class D from scratch. There are often many different specifications of the same class substitution, however, and in the following section we will see how easy it is to select a convenient one.

It is also for pragmatic reasons that we allow multiple, simultaneous substitutions. With this mechanism conflicting substitutions will cause compile-time errors; in contrast, a sequence of individual substitutions will always succeed but may yield an unexpected result. 


\subsection{Pragmatics}

The fact that class substitution realizes $\triangleleft_{S}$ is not sufficient to ensure that class substitution is a useful and pleasant programming mechanism. Only pragmatic arguments can really justify such a claim. In this section we attempt to give such arguments by showing some example programs which use class substitution, and by comparing class substitution with parameterized classes.

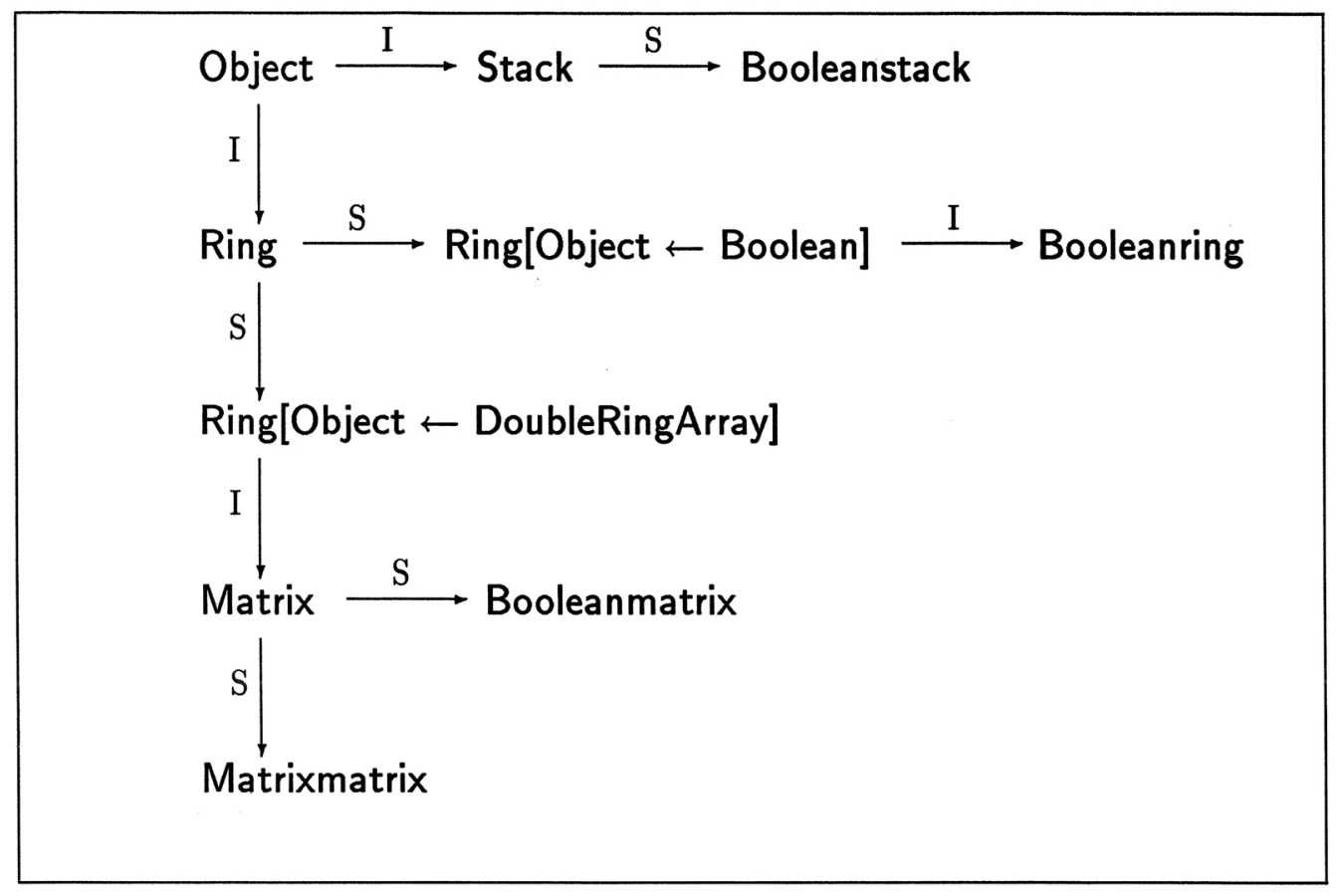

Figure 22: Programming with class substitution.

In figure 22 is shown a subclass hierarchy as it can be programmed using inheritance and class substitution. An arrow is labeled by "I" when the subclass is obtained by inheritance, and " $\mathrm{S}$ " if by class substitution. The detailed code for the classes will be given subsequently. We assume that the classes Boolean, Integer, and Array has been programmed already, and that Array instances respond to messages as specified in figure 23.

In the class Stack, the element type is Object, see figure 23. The classes Booleanstack and Integerstack are class substitutions of Stack. For example, 
Booleanstack is the class obtained from Stack by substituting all occurrences of Object by Boolean, including those in Array. Thus, Stack acts like a parameterized class but is just a class, not a second-order entity. This enables gradual generic-instantiations, as demonstrated in the following.

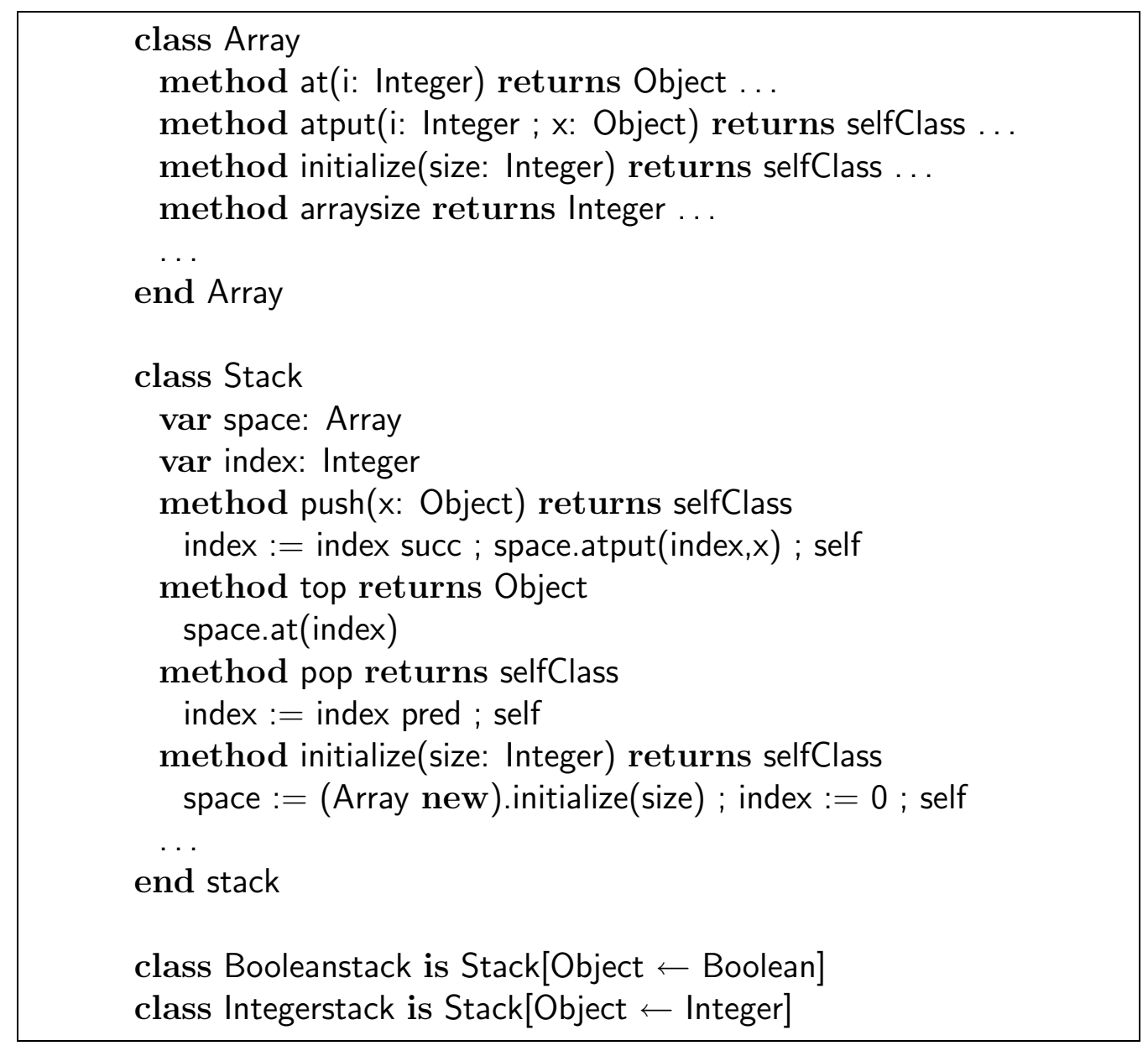

Figure 23: Stack classes.

The use of parameterized classes is the traditional approach to genericity $[38,39,49,34,48,42]$. Similar constructs are found in conventional procedural languages, for example ADA generic packages [24], and parameterized CLU clusters [35]. A parameterized class is a second-order entity which is generically-instantiated to specific classes when actual type parameters are supplied. Generic-instantiation of parameterized classes is less flexible than 
inheritance, since any class can be inherited but is not in itself parameterized. In other words, code reuse with parameterized classes requires planning; code reuse with inheritance does not.

In general, we will say that a genericity mechanism is a construct which allows the substitution of types in a class. Thus, class substitution is also a genericity mechanism, and in contrast to parameterized classes it is the orthogonal complement of inheritance. This indicates that class substitution gives more expressive power to an object-oriented language than parameterized classes, and indeed Meyer [38] argued that parameterized classes can be simulated by inheritance.

class Ring
var value: Object
method plus(other: selfClass) returns selfClass
self
method zerro returns selfClass
self
method getvalue returns Object
value
$\ldots$
end Ring
class BooleanRing inherits Ring[Object $\leftarrow$ Boolean]
method plus(other: selfClass) returns selfClass
value := value.or(other.getvalue) ; self
method zero returns selfClass
value := false ; self
$\ldots$
end BooleanRing

Figure 24: Ring classes.

Note that class substitution is not textual substitution; in general, textual substitution will not yield a statically correct subclass. For example, if we try to obtain Booleanstack by textually substituting occurrences of Object by Boolean, then among others the expression space.atput.(index,x) (in push) becomes statically incorrect; it will have an Boolean instance where an Object instance is required. For further examples, see [43]. 
Another drawback of parameterized classes is that they cannot be gradually generically-instantiated. This makes it awkward to, for example, declare a class Ring, then specialize it to a class Matrix, and finally specialize Matrix to a class Booleanmatrix. In the following we show how it can be done using inheritance and class substitution. The history of a class developed with inheritance and substitution can be thought of as an element of the language $(\mathrm{I}+\mathrm{S})^{*}$; in comparison, parameterized classes restrict the possible histories to S.I*.

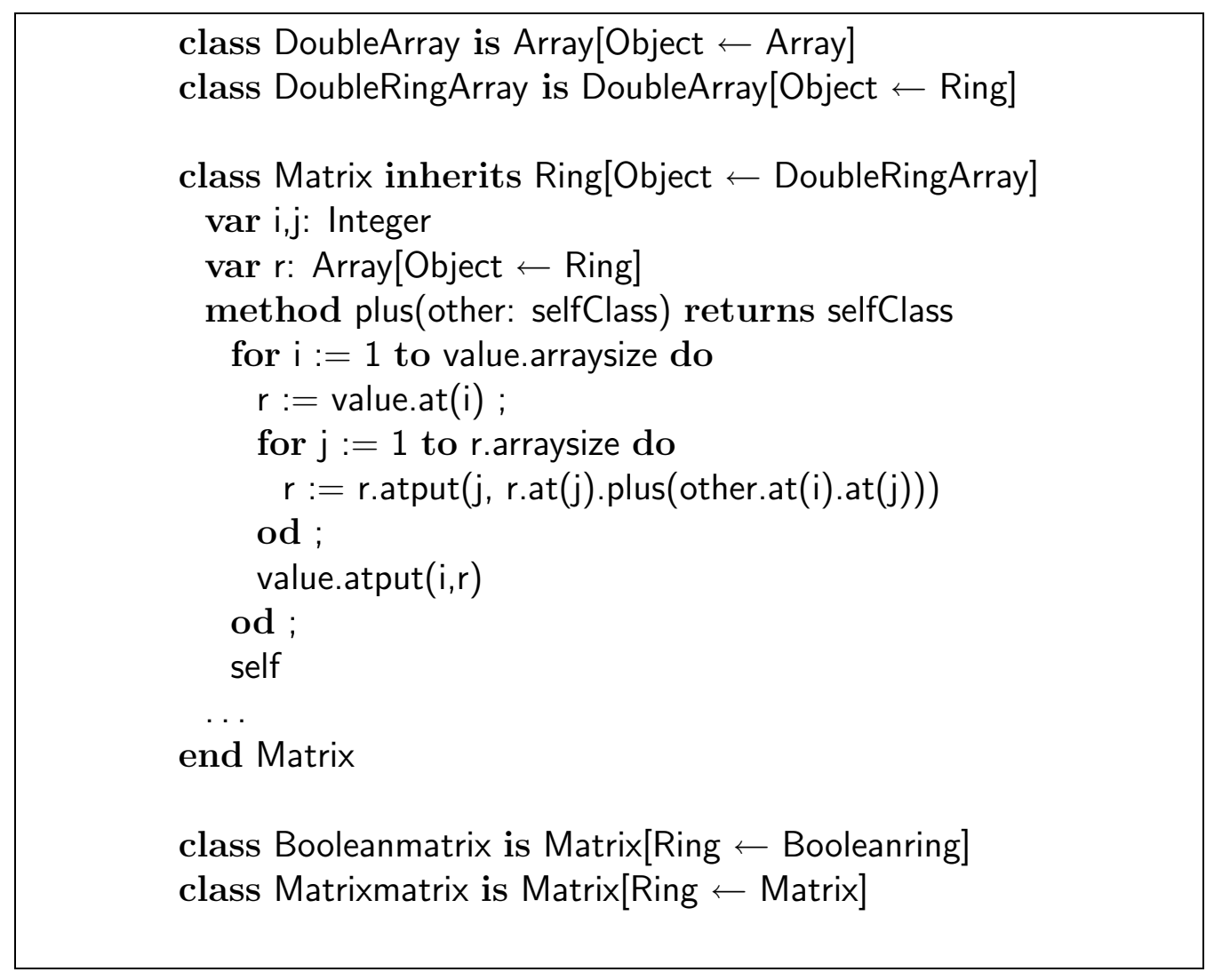

Figure 25: Matrix classes.

Consider the ring classes in figures 24 and 25. The class Booleanring inherits a class substitution of class Ring; thus, Booleanring is a subclass of Ring. This illustrates how class substitution and inheritance complement each other: first Object is substituted by Boolean; then the inherited procedures are implemented appropriately. This is further illustrated by the matrix classes, 
see figure 25. Again, the class Matrix is obtained through a class substitution followed by an application of inheritance. We take the liberty of using a for-statement, even though is has not been included in the syntax. Class Matrixmatrix is obtained through class substitution alone.

It seems that class substitution could solve the problems in the EIFFEL type system that were reported by Cook [15], since attributes cannot be redeclared in isolation in subclasses, there are no symmetries as with declaration by association, and generic-instantiation can be expressed as subclassing.

\section{Separate Compilation and Infinite Types}

One common aspect of many existing object-oriented languages has so far not been captured by our framework.

We have restricted our types to be finite sets. We argued that this was quite adequate, since in a given program any predicate could only be satisfied by finitely many classes.

With this type system we have developed a general notion of subclassing, under which any subclass can be implemented as an extension of its superclass. This is the fundamental idea of code reuse in object-oriented programming.

The concept of separate compilation, however, introduces a different level of code reuse, which does not fit as smoothly into our framework. When a class is separately compiled, then predicates cannot be expanded into finite sets, since only a part of the program is known at the time of compilation.

The traditional solution is to introduce a limited form of infinite sets; in particular, cones of the form

$$
\uparrow T=\{S \in \mathcal{U} \mid T \triangleleft S\}
$$

are employed. It is possible to generalize slightly: finite unions of cones and singletons can be used. The important restriction is that the sets can be finitely represented, and that membership and mutual inclusions are decidable. For cones, membership is just subclassing, and inclusion coincides with reverse subclassing, i.e.

$$
\uparrow S \subseteq \uparrow T \text { iff } T \triangleleft S
$$


A perfect match is not possible, but the types in a language such as BETA correspond closely to either singletons or cones [37].

Recall the important property of our framework that can be stated as the slogan: subclassing preserves subtyping. In the presence of cones, the picture changes dramatically. There exist classes $T \triangleleft S$ such that for some $\alpha, \beta$ we have

$$
\uparrow(T \downarrow \alpha) \subseteq \uparrow(T \downarrow \beta) \text { but } \uparrow(S \downarrow \alpha) \nsubseteq \uparrow(S \downarrow \beta)
$$

This unfortunately means that subclasses cannot be guaranteed to remain statically correct. A simple example is shown in figure 26, in which $\mathbf{S}$ is a statically incorrect subclass of the statically correct class $\mathrm{T}$.

\begin{tabular}{|l|}
\hline class $T$ \\
var $x: \uparrow$ Object \\
var $y: \uparrow$ Integer \\
method Assign returns selfClass \\
$x:=y ;$ self \\
end $T$ \\
class $S$ \\
var $x: \uparrow$ Object \\
var $y: \uparrow$ Integer \\
method Assign returns selfClass \\
$x:=y ;$ self \\
end $S$
\end{tabular}

Figure 26: A statically incorrect subclass.

There seem to be three possible solutions.

- restrict subclassing to preserve $\subseteq$ : unfortunately, only trivial subclasses can be allowed.

- restrict subtyping to be preserved by $\triangleleft$ : unfortunately, only trivial subtypes can be allowed.

- find a useful compromise between both subclassing and subtyping: unfortunately, no such compromise seems to be forthcoming. 
The situation does not look hopeful. The choice made by real-life languages is to keep both $\subseteq$ and $\triangleleft$, which leads to an statically unsound type system. The reactions to such a predicament fall on a spectrum ranging from $\mathrm{C}++$, in which these loop-holes are simply ignored, to BETA, in which the necessary run-time type-checks are inserted into the code of the superclass, yielding a dynamically sound type system.

We consider a more satisfactory solution to the problem of separate compilation to be an extremely hard challenge.

\section{Conclusion}

Our type system for object-oriented languages is conceptually simple and it ensures that programs are readable, reliable, and more efficient. Our subclassing order can be implemented straightforwardly and it contains inheritance and class substitution as an orthogonal basis.

It is too preliminary to judge the pragmatics of class substitution, but several examples indicate that it may be a useful alternative to parameterized classes. Future work includes implementation and experimentation with the mechanism.

The expansion algorithm for transforming a program that uses inheritance into one which does not may be of interest in itself. It can be useful for programmers who want to use inheritance but are required to implement their programs in a language which does not support it.

Acknowledgement. The authors thank Ole Lehrmann Madsen, Peter Mosses, and Flemming Nielson for helpful comments on drafts of the paper.

\section{References}

[1] Alfred V. Aho, John E. Hopcroft, and Jeffrey D. Ullman. The Design und Analysis of Computer Algorithms. Addison-Wesley Publishing Company, 1974. 
[2] Roberto M. Amadio and Luca Carcelli. Subtyping recursive types. In Eightteenth Symposium on Principles of Programming Languages. ACM Press, January 1991.

[3] Grady Booth. Object-Oriented Design with Applikations. The Benjamin/Cummings Publishing Company, 1991.

[4] Alan H. Borning and Daniel H. H. Ingalls. A type declaration and inference system for Smalltalk. In Ninth Symposium on Principles of Programming Languuges, pages 133-141. ACM Press, January 1982.

[5] Gilad Bracha and William Cook. Mixin-based inheritance. In Proc. OOPSLA/ECOOP'90, ACM SIGPLAN Fifth Annual Conference on Object-Oriented Programming Systems, Languages and Applications; European Conference on Object-Oriented Programming, 1990.

[6] Ronald J. Brachman. "I lied about the trees" or, defaults and definitions in knowledge representation. The AI Magazine, Fall, 1985.

[7] Kim B. Bruce. The equivalence of two semantic definitions for inheritance in object-oriented languages. In Proc. Mathmatical Foundations of Programming Semantics. Springer-Verlag (LNCS 442), 1991.

[8] Peter S. Canning, William R. Cook, Walter L. Hill, and Walter G. Olthoff. Interfaces for strongly-typed object-oriented programming. In Proc. OOPSLA'84, Fourth Annual Conference on Object-Oriented Programming Systems, Languages and Applications. ACM, 1989.

[9] L. Cardelli. A semantics of multiple inheritance. In G. Kahn, D. MacQueen, and Gordon Plotkin, editors, Semantics of Data Types, pages 51-68. Springer-Verlag (LNCS 173), 1984.

[10] L. Cardelli and P. Wegner. On understanding types, data abstraction, and polymorphism. ACM Computing Surveys, 17(4), December 1985.

[11] Luca Cardelli. Typeful programming. Technical report, Digital Equipment Corporation, 1989.

[12] Luca Cardelli and John C. Mitchell. Operations on records. In Proc. Mathmatical Foundations of Programming Semantics, pages 22-52. Springer-Verlag (LNCS 442), 1989. 
[13] Peter Coad and Edward Yourdon. Object-Oriented Analysis. Prentice Hall, 1991.

[14] W. R. Cook. A Denotational Semantics of Inheritance. PhD thesis, Brown University, 1989.

[15] William Cook. A proposal for making Eiffel type-safe. In Proc. ECOOP'89, European Conference on Object-Oriented Programming, 1989.

[16] William Cook, Walter Hill and Peter Canning. Inheritance is not subtyping. In Seventeenth Symposium on Principles of Programming Languages. ACM Press, January 1990.

[17] William Cook and Jens Palsberg. A denotational semantics of inheritance and its correctness. In Proc. OOPSLA'89, ACM SIGPLAN Fourth Annual Conference on Object-Oriented Programming Systems, Languages and Applications, 1989. To appear in Information and Computation.

[18] William R. Cook, Walter L. Hill, and Peter S. Canning. F-bounded polymorphism for object-oriented programming. In Proc. Conference on Functional Programming Languages and Computer Architecture, 1989.

[19] Bruno Courcelle. Infinite trees in normal form and recursive equations having a unique solution. Mathematical Systems Theory, 13:131-180, 1979 .

[20] Bruno Courcelle. Fundamental properties of infinite trees. Theoretical Computer Science, 25(1), 1983.

[21] Brad J. Cox. Object Oriented Programming, an Evolutionary Approach. Addison-Wesley Publishing Company, 1986.

[22] O. J. Dahl, B. Myhrhaug, and K. Nygaard. Simula 67 common base language. Technical report, Norwegian Computing Center, Oslo, Norway, 1968.

[23] Scott Danforth and Chris Tomlinson. Type theories and object-oriented programming. ACM Computing Surveys, 20(1), March 1988. 
[24] J. D. Ichbiah et al. Reference Manual for the Ada Programming Language. US DoD, July 1982.

[25] A. Goldberg and D. Robson. Smalkalk-80-The Language and its Implementation. Addison-Wesley, 1983.

[26] George Grätzer. General Lattice Theory. Birkhäuser, 1978.

[27] Justin O. Graver and RaIph E. Johnson. A type system for Smalltalk. In Seventeenth Symposium on Principles of Programming Languages, pages 136-150. ACM Press, January 1990.

[28] Justin Owen Graver. Type-Checking and Type-Inference for ObjectOriented Programming Languages. PhD thesis, Department of Computer Science, University of Illinois at Urbana-Champaign, August 1989. UIUCD-R-89-1539.

[29] R. E. Johnson. Type-checking Smalltalk. In Proc. OOPSLA'86, ObjectOriented Programming Systems, Languages and Applications. Sigplan Notices, 21(11), November 1986.

[30] Ralph E. Johnson and Brian Foote. Designing reuable classes. Journal of Object-Oriented Programming, June/July, 1988.

[31] S. Kamin. Inheritance in Smalltalk-80: A denotational definition. In Fifteenth Symposium on Principles of Programming Languages, pages 80-87. ACM Press, January 1988.

[32] B. B. Kristensen, O. L. Madsen, B. Møller-Pedersen, and K. Nygaard. The BETA programming language. In B. Shriver and P. Wegner, editors, Research Directions in Object-Oriented Programming, pages 7-48. MIT Press, 1987.

[33] B. B. Kristensen, O. L. Madsen, B. Møller-Pedersen, and K. Nygaard. Classification of actions or inheritance also for methods. In Proc. ECOOP'87, European Conference on Object-Oriented Programming. Springer-Verlag (LNCS 276), 1987.

[34] Karl J. Lieberherr and Arthur J. Riel. Contributions to teaching objectoriented design and programming. In Proc. OOPSLA'89, Fourth Annual Conference on Object-Oriented Programming Systems, Languages and Applications. ACM, 1989. 
[35] Barbara Liskov, Alan Snyder, Russell Atkinson, and Craig Scaffert. Abstraction mechanisms in CLU. Communications of the ACM, 20(8):564576, August 1977.

[36] Ole L. Madsen and Birger Møller-Pedersen. Virtual classes: A powerful mechanism in object-oriented programming. In Proc. OOPSLA'89, Fourth Annual Conference on Object-Oriented Programming Systems, Languages and Applications. ACM, 1989.

[37] Ole Lehrmann Madsen, Boris Magnusson, and Birger Møller-Pedersen. Strong typing of object-oriented languages revisited. In Proc. OOPSLA/ECOOP'90, ACM SIGPLAN Fifth Annual Conference on ObjectOriented Programming Systems, Languages and Applications; European Conference on Object-Oriented Programming, 1990.

[38] Bertrand Meyer. Genericity versus inheritance. In Proc. OOPSLA'86, Object-Oriented Programming Systems, Languages and Applications. Sigplan Notices, 21(11), November 1986.

[39] Bertrand Meyer. Object-Oriented Software Construction. Prentice-Hall, Englewood Cliffs, NJ, 1988.

[40] Robin Milner. A theory of type polymorphism in programming. Journal of Computer und System Sciences, 17, 1978.

[41] John C. Mitchell. Toward a typed foundation for method specialization and inheritance. In Seventeenth Symposium on Principles of Programming Languages. ACM Press, January 1990.

[42] Atsushi Ohori and Peter Buneman. Static type inference for parametric classes. In Proc. OOPSLA'89, Fourth Annual Conference on ObjectOriented Programming Systems, Languages and Applications. ACM, 1989.

[43] Jens Palsberg and Michael I. Schwartzbach. Type substitution for object-oriented programming. In Proc. OOPSLA/ECOOP'90, ACM SIGPLAN Fifth Annual Conference on Object-Oriented Programming Systems, Languages and Applications; European Conference on ObjectOriented Programming, 1990. 
[44] Jens Palsberg and Michael I. Schwartzbach. Object-oriented type inference. In Proc. OOPSLA'91, ACM SIGPLAN Sixth Annual Conference on Object-Oriented Programming Systems, Languages and Applications, 1991.

[45] Jens Palsberg and Michael I. Schwartzbach. What is type-safe code reuse? In Proc. ECOOP'91, Fifth European Conference on ObjectOriented Programming, 1991.

[46] Claus H. Pedersen. Extending ordinary inheritance schemes to include generalization. In Proc. OOPSLA'89, ACM SIGPLAN Fourth Annual Conference on Object-Oriented Programming Systems, Languages and Applications, 1989.

[47] U. S. Reddy. Objects as closures: Abstract semantics of object-oriented languages. In Proc. ACM Conference on Lisp and Functional Programming, pages 289-297. ACM, 1988.

[48] David Sandberg. An alternative to subclassing. In Proc. OOPSLA'86, Object-Oriented Programming Systems, Languages and Applications. Sigplan Notices, 21(11), November 1986.

[49] Craig Schaffert, Topher Cooper, Bruce Bullis, Mike Kilian, and Carrie Wilpolt. An introduction to Trellis/Owl. In Proc. OOPSLA'86, ObjectOriented Programming Systems, Languages and Applications. Sigplan Notices, 21(11), November 1986.

[50] Michael I. Schwartzbach. Static correctness of hierarchical procedures. In Proc. International Colloquium on Automata, Languages, and Programming 1990. Springer-Verlag (LNCS 443), 1990.

[51] Michael I. Schwartzbach. Type inference with inequalities. In Proc. TAPSOFT'91. Springer-Verlag (LNCS 493), 1991.

[52] A. Snyder. Inheritance and the development of encapsulated software components. In B. Shriver and P. Wegner, editors, Research Directions in Object-Oriented Programming. MIT Press, 1987.

[53] B. Stroustrup. The $C++$ Programming Language. Addison-Wesley, 1986. 
[54] David S. Touretzky. The Mathematics of Inheritance Systems. Morgan Kaufmann Publishers, 1986.

[55] P. Wegner. Dimensions of object-based language design. In Proc. OOPSLA'87, Object-Oriented Programming Systems, Languages and Applications, 1987.

[56] P. Wegner and S. B. Zdonik. Inheritance as an incremental modification mechanism or what like is and isn't like. In Proc. ECOOP'88, European Conference on Object-Oriented Programming. Springer-Verlag (LNCS 322), 1988.

[57] Niklaus Wirth. Programming in Modula-2. Springer-Verlag, New York, 1985. 